\title{
STRUCTURE OF THE GROUP PRESERVING A BILINEAR FORM
}

\author{
FERnANDo SzEchtman \\ Department of Mathematics \& Statistics, University of Regina, Saskatchewan, Canada, S4S 0A2 \\ e-mail: szechtf@math.uregina.ca
}

\begin{abstract}
We study the group of all linear automorphisms preserving an arbitrary bilinear form.
\end{abstract}

\section{Introduction}

Let $\varphi: V \times V \rightarrow F$ be a bilinear form on a finite dimensional vector space $V$ over a field $F$. We refer to the pair $(V, \varphi)$ as a bilinear space. The goal of this paper is to describe the structure of the group $G=G(V)$ of all linear automorphisms of $V$ preserving $\varphi$.

Classical groups such as the general linear, symplectic and orthogonal groups arise in this fashion. These classical cases have been thoroughly investigated (see [2], [8]). Arbitrary non-degenerate forms possess an asymmetry (as defined in [7]), which exerts a considerable influence on the structure of the bilinear space and associated group. This has recently been exploited by J. Fulman and R. Guralnick [4], where an array of useful information about $G$ is presented. The study of $G$ for a general bilinear form, possibly degenerate, over an arbitrary field does not seem to have been hitherto considered. The presence of a degenerate part enriches the structure of $G$, and it is in this regard that our main contribution takes place.

In general terms our approach consists of extracting structural information about $G$ by examining how $G$ acts on $V$ and its various $F G$-submodules.

Knowledge of the structure of $V$ as an $F G$-module will therefore be necessary. Our references in this regard will consist of the paper [7] by C. Riehm, its appendix [5] by P. Gabriel, and our recent article [3] with D. Djokovic.

An important decomposition of $V$ to be considered is

$$
V=V_{\text {odd }} \perp V_{\text {even }} \perp V_{\text {ndeg }},
$$


where $V_{\text {odd }}$, respectively $V_{\text {even }}$, is the orthogonal direct sum of indecomposable degenerate bilinear spaces of odd, respectively even, dimension, and $V_{\text {ndeg }}$ is non-degenerate. We identify $G\left(V_{\text {odd }}\right), G\left(V_{\text {even }}\right)$ and $G\left(V_{\text {ndeg }}\right)$ with subgroups of $G(V)$ by means of this decomposition.

As noted in [3], while $V_{\text {odd }}, V_{\text {even }}$ and $V_{\text {ndeg }}$ are uniquely determined by $V$ up to equivalence of bilinear spaces, they are not unique as subspaces of $V$, and in particular they are not $G$-invariant. Thus, one attempt to understand $G$ would consist of studying the structure of $G\left(V_{\text {odd }}\right), G\left(V_{\text {even }}\right)$ and $G\left(V_{\text {ndeg }}\right)$ separately, and then see how these groups fit together to form $G$.

This approach turns out to be fruitful, as we proceed to describe, with the notable exception of the structure of $G\left(V_{\text {ndeg }}\right)$ in the special case when the asymmetry of $V_{\text {ndeg }}$ is unipotent and the underlying field $F$ has characteristic 2. This is what C. Riehm refers to as Case IIb in his paper.

We begin our journey in section 2 by establishing notation and terminology. Two important $G$-invariant subspaces of $V$ described here are what we denote by $V_{\infty}$ and ${ }^{\infty} V$ in [3]. Briefly, any choice for $V_{\text {odd }}$ will contain $V_{\infty}$, which is in fact the only totally isotropic subspace of $V_{\text {odd }}$ of maximum possible dimension; whatever the choices for $V_{\text {even }}$ and $V_{\text {ndeg }}$, it turns out that ${ }^{\infty} V=V_{\infty} \perp V_{\text {even }} \perp V_{\text {ndeg }}$.

Section 3 contains basic tools regarding $V$ and $G$ to be used throughout the paper. An important feature of this section is the introduction -in Definition 3.17- of a family of 1-parameter subgroups of $G\left(V_{\text {odd }}\right)$ which will play a decisive role in shedding light on the structure of both $G\left(V_{\text {odd }}\right)$ and $G$.

The actual paper can be said to begin in section 4 . We first introduce a normal subgroup $N$ of $G$, defined as the intersection of various pointwise stabilizers in $G$ when it acts on certain sections of the $F G$-module $V$. It is shown in Theorem 4.4 that $G=N \rtimes E$, where $E \cong \prod_{1 \leq i \leq t} \mathrm{GL}_{m_{i}}(F)$ and the parameters $t$ and $m_{1}, \ldots, m_{t}$ depend only on $V$, as explained below. We have

$$
V_{\text {odd }}=V_{1} \perp V_{2} \perp \cdots \perp V_{t},
$$

where each $V_{i}$ is the orthogonal direct sum of $m_{i}$ bilinear subspaces, each of which is 
isomorphic to a Gabriel block of size $2 s_{i}+1$, with $s_{1}>s_{2}>\cdots>s_{t}$. Here by a Gabriel block of size $r \geq 1$ we mean the only indecomposable degenerate bilinear space of dimension $r$, up to equivalence, namely one that admits a nilpotent Jordan block of size $r$ as its Gram matrix.

As a byproduct of the results in this section we are able to describe the irreducible constituents of the $F G$-submodule $V_{\infty}$ of $V$. This is taken up in section 5 (Theorem 5.1). These constituents are seen later in section 6 to be intimately connected to certain $F G$-modules arising as sections of $G$ itself. The end of this section also gives a second decomposition for $G$, namely

$$
G(V)=G\left[{ }^{\infty} V / V_{\infty}\right] \rtimes\left(G\left(V_{\text {even }}\right) \times G\left(V_{\text {ndeg }}\right)\right)
$$

where in general $G[Y]$ denotes the pointwise stabilizer of $G$ acting on a $G$-set $Y$.

Section 6 goes much deeper than previous sections. In view of the decomposition $G=$ $N \rtimes E$ and the clear structure of $E$, our next goal is to study $N$ and the action of $G$ upon it. In Theorem 6.10 we prove $N=G\left[V_{\infty}\right] \rtimes U$, where $U$ is a unipotent subgroup of $G\left(V_{\text {odd }}\right)$ generated by the 1-parameter subgroups referred to above. An extensive analysis of the nilpotent group $N / G\left[V_{\infty}\right]$ is carried out. First of all, its nilpotency class is seen in Theorem 6.2 to be $t-1$. As a nilpotent group $N / G\left[V_{\infty}\right]$, possesses a descending central series. We actually produce in Theorem 6.22 a $G$-invariant descending central series for $N / G\left[V_{\infty}\right]$ each of whose factors has a natural structure of $F G$-module, and irreducible at that. These irreducible $F G$-modules are in close relationship with the irreducible constituents of the $F G$-module $V_{\infty}$. By taking into account all factors in our series we deduce a formula for the dimension of $U$, in the algebraic/geometric sense, which turns out to be equal to the number of 1-parameter groups generating $U$ and referred to above. Theorem 6.20 proves

$$
\operatorname{dim} U=\sum_{1 \leq i<j \leq t}\left(s_{i}-s_{j}+1\right) m_{i} m_{j}
$$

As a byproduct of the results in this section we also obtain in Theorem 6.15 the irreducible constituents of the $F G$-module $V /{ }^{\infty} V$, which in turn are closely related to other FG-modules also arising as sections of $G$. 
Section 7 concentrates on the next logical target, namely $G\left[V_{\infty}\right]$. We know from above that $G=N \rtimes E$ and $N=G\left[V_{\infty}\right] \rtimes U$. Here we prove (Theorem 7.1) that $U$ actually normalizes $E$-so $G=G\left[V_{\infty}\right] \rtimes(U \rtimes E)$-, that $G\left[V_{\infty}\right]$ admits the decomposition $G\left[V_{\infty}\right]=\left(G\left[V_{\infty}\right] \cap G\left[^{\infty} V / V_{\infty}\right]\right) \rtimes\left(G\left(V_{\text {even }}\right) \times G\left(V_{\text {ndeg }}\right)\right)$, and that $U \rtimes E$ actually commutes with $G\left(V_{\text {even }}\right) \times G\left(V_{\text {ndeg }}\right)$ elementwise. We thus obtain the important decomposition

$$
G=\left(G\left[V_{\infty}\right] \cap G\left[{ }^{\infty} V / V_{\infty}\right]\right) \rtimes\left(G\left(V_{\text {even }}\right) \times G\left(V_{\text {ndeg }}\right) \times(U \rtimes E)\right)
$$

With the structure of $U \rtimes E$ already clarified, the next step consists of studying $G\left(V_{\text {even }}\right)$ and $G\left(V_{\text {ndeg }}\right)$ on their own, and see what is the structure of $G\left[V_{\infty}\right] \cap G\left[{ }^{\infty} V / V_{\infty}\right]$.

Note that $G\left(V_{\text {odd }}\right)$ seems to be absent above. But that is only an illusion, which is clarified in section 11. In fact, $G\left(V_{\text {odd }}\right)$ is essentially what is holding the above decomposition together. If $V_{\text {odd }}=(0)$ the $V_{\text {even }}$ and $V_{\text {ndeg }}$ are in fact $G$-invariant, as [3] shows, so $G=G\left(V_{\text {even }}\right) \times G\left(V_{\text {ndeg }}\right)$.

Section 8 begins by laying the foundations (Theorem 8.3) for a combined attack on $G\left(V_{\text {even }}\right)$ and certain direct factors of $G\left(V_{\text {ndeg }}\right)$. Theorem 8.5 then exploits this by describing $G\left(V_{\text {even }}\right)$ as the centralizer of a nilpotent element of known similarity type in the general linear group.

Attention in section 9 is focused on $G\left(V_{\mathrm{ndeg}}\right)$. This group is approached via the study of $V_{\text {ndeg }}$ as a module over the polynomial algebra $F[t]$ by means of the asymmetry of $\left.\varphi\right|_{V_{\text {ndeg }}}$, as outlined in [7]. Thus (see equation (28)) $G\left(V_{\text {ndeg }}\right)$ is isomorphic to the direct product of groups of the form $G(W)$, where $W$ is a non-degenerate bilinear space whose type, according to C. Riehm, is either I, IIa or IIb.

In the first case $G(W)$ is seen (in Theorem 9.1 via Theorem 8.3) to be isomorphic to the centralizer in a general linear group of a linear automorphism of known similarity type. If $F$ is algebraically closed this linear automorphism can be replaced by a nilpotent endomorphism.

Case IIa is more difficult. We find (Theorem 9.6) $G(W)$ to be equal to the centralizer in a symplectic or orthogonal group of a particular linear endomorphism. If $F$ has characteristic not 2 this element is in the corresponding symplectic or orthogonal Lie algebra. If in addition $F$ is algebraically closed we can ensure (Theorems 9.8 and 9.9) that this 
element is nilpotent of known similarity class. These centralizers are described in various places, e.g. in $[6,10]$.

As mentioned already above the case when the asymmetry of $V_{\text {ndeg }}$ is unipotent and $F$ has characteristic 2, i.e. Case IIb, remains unsolved.

Section 10 concentrates on $G\left[V_{\infty}\right] \cap G\left[^{\infty} V / V_{\infty}\right]$. One sees rather rapidly (Lemmas 10.1, 10.2 and 10.4) that $G\left[V_{\infty}\right] \cap G\left[{ }^{\infty} V / V_{\infty}\right]$ is unipotent of nilpotency of class $\leq 2$ having $\left.G^{\infty} V\right]$ in its center, the corresponding quotient group being abelian. Thus the study of $G\left[V_{\infty}\right] \cap G\left[{ }^{\infty} V / V_{\infty}\right]$ is divided into that of $G\left[{ }^{\infty} V\right]$ and $G\left[V_{\infty}\right] \cap G\left[{ }^{\infty} V / V_{\infty}\right] / G\left[{ }^{\infty} V\right]$.

Well, $G\left[{ }^{\infty} V\right]$ is naturally an $F G$-module and, much as in section 6, we find (Theorem 10.18) its irreducible constituents and explain how they relate to those of $V /{ }^{\infty} V$. As in section 6, this requires considerable amount of work. In particular, the dimension of $G\left[{ }^{\infty} V\right]$ is found. We also compute (Proposition 10.20) the dimension of the quotient group $G\left[V_{\infty}\right] \cap G\left[^{\infty} V / V_{\infty}\right] / G\left[^{\infty} V\right]$, thereby obtaining (Theorem 10.21) a formula for the dimension of $G\left[V_{\infty}\right] \cap G\left[{ }^{\infty} V / V_{\infty}\right]$, which reads

$$
\operatorname{dim} G\left[V_{\infty}\right] \cap G\left[{ }^{\infty} V / V_{\infty}\right]=\operatorname{dim}\left(V / V_{\infty}\right) \times\left(m_{1}+\cdots+m_{t}\right)
$$

Section 11 furnishes a few more decompositions for $G$ and $G\left(V_{\text {odd }}\right)$ (Theorems 11.1 and 11.2) and includes an example (Theorem 11.3) on the structure of $G\left(V_{\text {odd }}\right)$ in a special but interesting case. The structure of $G\left(V_{\text {odd }}\right)$ is fully revealed in this case.

Our last section makes some comments on an alternative approach to the study of $G$.

A few words about the origin of this paper are in order. After our joint work [3] with D. Djokovic, we were excited about the prospect of being able to attack the present problem. We worked rather intensively together for quite some time in fruitful collaboration. Each of us built his own version of the paper, and at one point our methods and some of our goals became too far apart for us to be able to amalgamate them into a single paper. Even though we agreed to submit our versions separately, the outcome of this project should be regarded as joint work. 


\section{Generalities}

Let $F$ be a field. A bilinear space over $F$ is a pair $(V, \varphi)$, where $V$ is a finite dimensional $F$-vector space and $\varphi: V \times V \rightarrow F$ is a bilinear form. An isometry from a bilinear space $\left(V_{1}, \varphi_{1}\right)$ to a bilinear space $\left(V_{2}, \varphi_{2}\right)$ is a linear isomorphism $g: V_{1} \rightarrow V_{2}$ satisfying

$$
\varphi_{2}(g v, g w)=\varphi_{1}(v, w), \quad v, w \in V_{1} .
$$

Two bilinear spaces are equivalent if there exists an isometry between them. The isometry group of a bilinear space $(V, \varphi)$ is the group of all isometries from $(V, \varphi)$ into itself.

We henceforth fix a bilinear space $(V, \varphi)$. Its isometry group will be denoted by $G(V, \varphi)$, $G(V), G(\varphi)$, or simply by $G$. Explicit reference to $\varphi$ will be omitted when no confusion is possible. We shall often write $\langle v, w\rangle$ instead of $\varphi(v, w)$.

The space of all bilinear forms on $V$ will be denoted by $\operatorname{Bil}(V)$. There is an action of $\mathrm{GL}(V)$ on $\operatorname{Bil}(V)$ given by

$$
(g \cdot \phi)(v, w)=\phi\left(g^{-1} v, g^{-1} w\right), \quad g \in \mathrm{GL}(V), \phi \in \operatorname{Bil}(V), v, w \in V .
$$

Thus the isometry group of $(V, \varphi)$ is the stabilizer of $\varphi$ under this action.

If $U$ is a subspace of $V$, then $U$ becomes a bilinear space by restricting $\varphi$ to $U \times U$. We write $V=U \perp W$ if $V=U \oplus W$ and $\langle U, W\rangle=\langle W, U\rangle=0$. In this case we refer to $U$ and $W$ as orthogonal summands of $V$. A bilinear space is indecomposable if it lacks proper non-zero orthogonal summands. If $\langle U, U\rangle=0$ then $U$ is totally isotropic.

For a subspace $U$ of $V$, let

$$
L(U)=\{v \in V \mid\langle v, U\rangle=0\}, \quad R(U)=\{v \in V \mid\langle U, v\rangle=0\} .
$$

Here $L(V)$ and $R(V)$ are the left and right radicals of $V$, and $\operatorname{Rad}(V)=L(V) \cap R(V)$ is the radical of $V$. We have $\operatorname{dim} L(V)=\operatorname{dim} R(V)$, and we say that $V$ is non-degenerate whenever this number is 0 . Otherwise $V$ is degenerate. A degenerate space is totally degenerate if all its non-zero orthogonal summands are degenerate.

We view $L$ and $R$ as operators which assign to each subspace of $V$ its left and right orthogonal complements, respectively. If required we will write $L_{V}$ and $R_{V}$ for them. We 
may compound these operators, denoting by $L^{i}$ and $R^{i}$ their respective $i$-th iterates. By convention, $L^{0}$ and $R^{0}$ are the identity operators. By definition

$$
L(V) \subseteq L^{3}(V) \subseteq L^{5}(V) \subseteq \cdots \subseteq L^{4}(V) \subseteq L^{2}(V) \subseteq L^{0}(V)=V
$$

and similarly for $R$. We denote by $L_{\infty}(V), R_{\infty}(V), L^{\infty}(V)$ and $R^{\infty}(V)$ the subspaces of $V$ at which the sequences $\left(L^{2 k+1}(V)\right)_{k \geq 0},\left(R^{2 k+1}(V)\right)_{k \geq 0},\left(L^{2 k}(V)\right)_{k \geq 0}$ and $\left(R^{2 k}(V)\right)_{k \geq 0}$ stabilize, respectively. We set $V_{\infty}=L_{\infty}(V)+R_{\infty}(V)$ and ${ }^{\infty} V=L^{\infty}(V)+R^{\infty}(V)$. By construction both $V_{\infty}$ and ${ }^{\infty} V$ are $G$-invariant.

For $r \geq 1$ and $\lambda \in F$, denote by $J_{r}(\lambda)$ the lower Jordan block of size $r$ corresponding to the eigenvalue $\lambda$. Thus

$$
J_{1}(\lambda)=(\lambda), \quad J_{2}(\lambda)=\left(\begin{array}{cc}
\lambda & 0 \\
1 & \lambda
\end{array}\right), \quad J_{3}(\lambda)=\left(\begin{array}{ccc}
\lambda & 0 & 0 \\
1 & \lambda & 0 \\
0 & 1 & \lambda
\end{array}\right), \ldots
$$

Write $N_{r}$ for a bilinear space whose underlying form has matrix $J_{r}(0)$ relative to some basis. We shall refer to the bilinear space $N_{r}$ as a Gabriel block and to $r$ as its size. We refer the reader to $[3,12]$ for the following formulation of a theorem due to P. Gabriel [5].

2.1 Theorem Let $(V, \varphi)$ be a bilinear space over $F$. Then

(a) $V=V_{\text {tdeg }} \perp V_{\text {ndeg }}$, where $V_{\text {tdeg }}$ is the orthogonal direct sum of Gabriel blocks and $V_{\text {ndeg }}$ is non-degenerate (either of them possibly 0 ).

(b) The sizes and multiplicities of the Gabriel blocks appearing in $V_{\text {tdeg }}$ are uniquely determined by $V$.

(c) The equivalence class of $V_{\text {ndeg }}$ is uniquely determined by $V$.

(d) Up to equivalence, the only indecomposable and degenerate bilinear space of dimension $r \geq 1$ is $N_{r}$.

We refer to $V_{\mathrm{tdeg}}$ and $V_{\mathrm{ndeg}}$ as the totally degenerate and non-degenerate parts of $V$, respectively. We may write $V_{\text {tdeg }}=V_{\text {even }} \perp V_{\text {odd }}$, where $V_{\text {even }}$ resp. $V_{\text {odd }}$ is the orthogonal direct sum of Gabriel blocks of even resp. odd size. We refer to them as the even and odd parts of $V$. 
We fix a decomposition

$$
V=V_{\text {odd }} \perp V_{\text {even }} \perp V_{\text {ndeg }},
$$

and identify $G\left(V_{\text {odd }}\right), G\left(V_{\text {even }}\right)$ and $G\left(V_{\text {ndeg }}\right)$ with their image in $G(V)$, obtained by extending via the identity on the complements exhibited in (1).

While none of $V_{\text {even }}, V_{\text {odd }}, V_{\text {ndeg }}$ are in general $G$-invariant (see [3]) we know from [3] that, whatever the choices for these are, $V_{\infty}$ is the only totally isotropic subspace of $V_{\text {odd }}$ of maximum dimension and

$$
{ }^{\infty} V=V_{\infty} \perp V_{\text {even }} \perp V_{\text {ndeg }}
$$

2.1 Notation If $G$ acts on a set $X$ and $Y \subseteq X$ then $G[Y]$ and $G\{Y\}$ denote the pointwise and global stabilizers of $Y$ in $G$, respectively.

2.2 Notation If $Y$ is a subset of $G$ then $\langle Y\rangle$ denotes the subgroup of $G$ generated by $Y$.

2.3 Notation If $W$ is an $F$-vector space and $f_{1}, \ldots, f_{m}$ are vectors in $W$ then their span will be denoted by $\left(f_{1}, \ldots, f_{m}\right)$.

2.4 Notation The transpose of $\phi \in \operatorname{Bil}(V)$ is the bilinear form $\phi^{\prime} \in \operatorname{Bil}(V)$, defined by

$$
\phi^{\prime}(v, w)=\phi(w, v), \quad v, w \in V .
$$

The transpose of a matrix $A$ will be denoted by $A^{\prime}$.

\section{Lemmata}

We fix a decomposition

$$
V_{\text {odd }}=V_{1} \perp V_{2} \perp \cdots \perp V_{t},
$$

where each $V_{i}$ is the orthogonal direct sum of $m_{i}$ bilinear subspaces, each of which is isomorphic to a Gabriel block of size $2 s_{i}+1$, with $s_{1}>s_{2}>\cdots>s_{t}$. By means of the decomposition (3) we may identify each $G\left(V_{i}\right)$ with its image in $G\left(V_{\text {odd }}\right)$. 
We have

$$
V_{i}=V^{i, 1} \perp V^{i, 2} \perp \cdots \perp V^{i, m_{i}}
$$

where each $V^{i, p}, 1 \leq p \leq m_{i}$, has a basis

$$
e_{1}^{i, p}, \ldots, e_{2 s_{i}+1}^{i, p}
$$

relative to which the matrix of $\varphi$ is equal to $J_{2 s_{i}+1}(0)$. We shall consider the basis $\mathcal{B}$ of $V_{\text {odd }}$, defined by

$$
\mathcal{B}=\left\{e_{k}^{i, p} \mid 1 \leq i \leq t, 1 \leq p \leq m_{i}, 1 \leq k \leq 2 s_{i}+1\right\}
$$

For $1 \leq i \leq t$ let $V_{i}^{\dagger}$ be the span of $e_{2 k}^{i, p}, 1 \leq p \leq m_{i}$ and $1 \leq k \leq s_{i}$, and let

$$
V_{\text {odd }}^{\dagger}=\underset{1 \leq i \leq t}{\oplus} V_{i}^{\dagger}
$$

Note that $V_{\text {odd }}^{\dagger}$ is a totally isotropic subspace of $V_{\text {odd }}$ satisfying

$$
V_{\text {odd }}=V_{\infty} \oplus V_{\text {odd }}^{\dagger}
$$

There is no loss of generality in considering this particular subspace, as shown in Lemma 3.3 below.

3.1 Lemma $G\left[V_{\infty}\right] \subseteq G\left[V /{ }^{\infty} V\right]$

Proof. Let $g \in G\left[V_{\infty}\right], x \in V$ and $y \in V_{\infty}$. Then

$$
\langle x-g x, y\rangle=\langle x, y\rangle-\langle g x, y\rangle=\langle x, y\rangle-\left\langle x, g^{-1} y\right\rangle=\langle x, y\rangle-\langle x, y\rangle=0 \text {. }
$$

Since $L\left(V_{\infty}\right)={ }^{\infty} V$, the result follows.

3.2 Lemma $G\left[{ }^{\infty} V\right]=G\left(V_{\text {odd }}\right) \cap G\left[V_{\infty}\right] \subseteq G\left[V / V_{\infty}\right]$

Proof. Since

$$
L\left(V_{\text {even }} \oplus V_{\text {ndeg }}\right) \cap R\left(V_{\text {even }} \oplus V_{\text {ndeg }}\right)=V_{\text {odd }},
$$

we have $G\left[^{\infty} V\right] \subseteq G\left(V_{\text {odd }}\right)$. For $g \in G\left[^{\infty} V\right]$ and $v \in V_{\text {odd }}$, by Lemma 3.1 we have

$$
g v-v \in{ }^{\infty} V \cap V_{\text {odd }}=V_{\infty} .
$$

Hence $G\left[{ }^{\infty} V\right] \subseteq G\left[V / V_{\infty}\right]$. 
3.3 Lemma The permutation action of $G\left(V_{\text {odd }}\right)$ on the set of totally isotropic subspaces $W$ of $V_{\text {odd }}$ satisfying $V_{\text {odd }}=V_{\infty} \oplus W$ is transitive. In fact, restriction to $\left.G{ }^{\infty} V\right]$ yields a regular action.

Proof. Let $W$ and $W^{\prime}$ be totally isotropic subspaces of $V_{\text {odd }}$ complementing $V_{\infty}$. By Lemma 3.1 we have

$$
G\left[^{\infty} V\right] \cap G\{W\}=G\left[^{\infty} V\right] \cap G\left[V /{ }^{\infty} V\right] \cap G\{W\}=<1>
$$

We next show the existence of $g \in G\left[^{\infty} V\right]$ satisfying $g(W)=W^{\prime}$. The decomposition $V_{\text {odd }}=V_{\infty} \oplus W^{\prime}$ gives rise to a unique projection $p \in \operatorname{End}_{F}\left(V_{\text {odd }}\right)$ with image $W^{\prime}$ and kernel $V_{\infty}$. Define $g \in \mathrm{GL}\left(V_{\text {odd }}\right)$ by

$$
g(v+w)=v+p(w), \quad v \in V_{\infty}, w \in W
$$

Let $u, v \in V_{\infty}$ and $w, z \in W$. Since $V_{\infty}, W$ and $W^{\prime}$ are totally isotropic, and $(p-1) V_{\text {odd }} \subseteq$ $V_{\infty}$, we have

$$
\begin{aligned}
\langle g(u+w), g(v+z)\rangle & =\langle u+p w, v+p z\rangle=\langle u, p z\rangle+\langle p w, v\rangle \\
& =\langle u,(p-1) z+z\rangle+\langle(p-1) w+w, v\rangle=\langle u, z\rangle+\langle w, v\rangle \\
& =\langle u+w, z\rangle+\langle u+w, v\rangle=\langle u+w, v+z\rangle .
\end{aligned}
$$

Then $g \in G\left(V_{\text {odd }}\right)$ fixes $V_{\infty}$ elementwise and sends $W$ to $W^{\prime}$, which completes the proof.

3.4 Lemma Let $W=N_{2 s+1}$ be a Gabriel block of odd size $2 s+1$. Let $f_{1}, \ldots, f_{2 s+1}$ be a basis of $W$ relative to which the underlying bilinear form has basis $J_{2 s+1}(0)$.

(a) If $0 \leq k \leq s$ then

$L^{2 k+1}(W)=\left(f_{1}, f_{3}, f_{5}, \ldots, f_{2 k+1}\right)$ and $R^{2 k+1}(W)=\left(f_{2 s+1}, f_{2 s-1}, f_{2 s-3}, \ldots, f_{2(s-k)+1}\right)$.

(b) If $k \geq s$ then

$$
L^{2 k+1}(W)=R^{2 k+1}(W)=W_{\infty}=\left(f_{1}, f_{3}, f_{5}, \ldots, f_{2 s+1}\right) .
$$

Proof. This follows easily from the definition of the operators $L$ and $R$. 
3.5 Lemma Let $W=N_{2 s}$ be a Gabriel block of even size $2 s$. Let $f_{1}, \ldots, f_{2 s}$ be a basis of $W$ relative to which the underlying bilinear form has basis $J_{2 s}(0)$.

(a) If $0 \leq k \leq s-1$ then

$$
L^{2 k+1}(W)=\left(f_{1}, f_{3}, f_{5}, \ldots, f_{2 k+1}\right) \text { and } R^{2 k+1}(W)=\left(f_{2 s}, f_{2 s-2}, f_{2 s-4}, \ldots, f_{2(s-k)}\right) .
$$

(b) If $k \geq s-1$ then

$L^{2 k+1}(W)=\left(f_{1}, f_{3}, f_{5}, \ldots, f_{2 s-1}\right)=L_{\infty}(W), R^{2 k+1}(W)=\left(f_{2 s}, f_{2 s-2}, f_{2 s-4}, \ldots, f_{2}\right)=R_{\infty}(W)$

(c) $W=L_{\infty}(W) \oplus R_{\infty}(W)$.

Proof. (a) and (b) follow easily from the definition of the operators $L$ and $R$, and (c) is consequence of (b).

3.6 Lemma If $V=U \perp W$ then

$$
L_{V}^{k}(V)=L_{U}^{k}(U) \perp L_{W}^{k}(W) \text { and } R_{V}^{k}(V)=R_{U}^{k}(U) \perp R_{W}^{k}(W), \quad k \geq 1 .
$$

Proof. This follows easily from the definition of the operators $L$ and $R$.

3.7 Lemma $\quad$ Let $1 \leq i \leq t$ and $0 \leq k, l$.

(a) If $k, l \leq s_{i}$. Then a basis for $L^{2 k+1}(V) \cap R^{2 l+1}(V) \cap V_{i}$ is formed by all $e_{2 c+1}^{i, p}$, if any, such that $1 \leq p \leq m_{i}$ and $s_{i}-l \leq c \leq k$.

(b) If $k>s_{i}$ (resp. $l>s_{i}$ ) then a basis for $L^{2 k+1}(V) \cap R^{2 l+1}(V) \cap V_{i}$ is formed by all $e_{2 c+1}^{i, p}$ such that $1 \leq p \leq m_{i}, 0 \leq c \leq s_{i}$, and $s_{i}-l \leq c($ resp. $c \leq k)$.

Proof. This follows from Lemmas 3.4 and 3.6 by means of the decompositions (1), (3) and (4).

3.8 Lemma Let $k, l \geq 0$ and $1 \leq i \leq t$. Then

$$
L^{2 k+1}(V) \cap R^{2 l+1}(V) \cap V_{i} \neq(0)
$$

if and only if $k+l \geq s_{i}$.

Proof. This follows from Lemma 3.7. 
3.9 Lemma Let $1 \leq i \leq t$ and $0 \leq j, k$. Suppose $i+j \leq t$ and $k \leq s_{i}$. Then

$$
L(V) \cap R^{2\left(s_{i}-k\right)+1}(V) \cap V_{i+j} \neq(0)
$$

if and only if $s_{i}-k \geq s_{i+j}$.

Proof. This is a particular case of Lemma 3.8.

3.10 Lemma Let $1 \leq i \leq t$ and $0 \leq k \leq s_{i}$. Then

$$
L^{2 k+1}(V) \cap R^{2\left(s_{i}-k\right)+1}(V) \cap V_{i}=\left(e_{2 k+1}^{i, 1}, \ldots, e_{2 k+1}^{i, m_{i}}\right) .
$$

Proof. This is a particular case of Lemma 3.7.

3.11 Definition Consider the subspaces of $V_{\infty}$ defined as follows:

$$
V(i)=\underset{i \leq j \leq t}{\oplus}\left(V_{j}\right)_{\infty}, \quad 1 \leq i \leq t
$$

and set $V(i)=0$ for $i>t$.

3.12 Lemma If $1 \leq i \leq t$ then

$$
V(i)=\sum_{0 \leq k \leq s_{i}} L^{2 k+1}(V) \cap R^{2\left(s_{i}-k\right)+1}(V) .
$$

Proof. By virtue of Lemmas 3.5, 3.6 and 3.8, and the decompositions (1), (3) and (4) it follows that the right hand side is contained in $V(i)$. By Lemma 3.10, if $i \leq j \leq t$ then

$$
\left(V_{j}\right)_{\infty}=\sum_{0 \leq k \leq s_{j}} L^{2 k+1}(V) \cap R^{2\left(s_{j}-k\right)+1}(V) \cap V_{j} \subseteq \sum_{0 \leq k \leq s_{i}} L^{2 k+1}(V) \cap R^{2\left(s_{i}-k\right)+1}(V),
$$

as required.

3.13 Lemma The subspaces $V(i)$ are $G$-invariant.

Proof. This follows from Lemma 3.12 .

3.14 Lemma Let $W=N_{2 s+1}$ be a Gabriel block of odd size $2 s+1$. Let $f_{1}, \ldots, f_{2 s+1}$ be a basis of $W$ relative to which the underlying bilinear form, say $\phi$, has basis $J_{2 s+1}(0)$. Then $\operatorname{Rad}\left(\phi-\phi^{\prime}\right)=\left(f_{1}+f_{3}+\cdots+f_{2 s-1}+f_{2 s+1}\right)$. 
Proof. Clearly the vector $f_{1}+f_{3}+\cdots+f_{2 s-1}+f_{2 s+1}$ belongs to the radical of $\phi-\phi^{\prime}$. Since the nullity of the matrix $J_{2 s+1}(0)-J_{2 s+1}(0)^{\prime}$ is equal to one, the result follows.

3.15 Notation For each $1 \leq i \leq t$ and each $1 \leq p \leq m_{i}$ let

$$
E^{i, p}=e_{1}^{i, p}+e_{3}^{i, p}+\cdots+e_{2 s_{i}+1}^{i, p} .
$$

3.16 Lemma If $1 \leq i \leq t$ then

$$
\operatorname{Rad}\left(\varphi-\varphi^{\prime}\right) \cap V_{i}=\left(E^{i, 1}, \ldots, E^{i, m_{i}}\right) .
$$

Proof. By Lemma 3.14 we have

$$
\operatorname{Rad}\left(\varphi-\varphi^{\prime}\right) \cap V_{i}=\underset{1 \leq p \leq m_{i}}{\oplus} \operatorname{Rad}\left(\varphi-\varphi^{\prime}\right) \cap V^{i, p}=\underset{1 \leq p \leq m_{i}}{\oplus}\left(E^{i, p}\right)=\left(E^{i, 1}, \ldots, E^{i, m_{i}}\right) .
$$

3.17 Definition Let $1 \leq i ; 0 \leq k, j ; 1 \leq p, q$. Suppose $i+j \leq t ; k \leq s_{i}-s_{i+j}$; $1 \leq p \leq m_{i} ; 1 \leq q \leq m_{i+j} ; p \neq q$ if $j=0$. Consider the 1-parameter subgroup of $G\left(V^{i, p} \perp V^{i+j, q}\right)$-or simply $G\left(V^{i, p}\right)$ if $j=0$ - formed by all $g_{2 k+1, y}^{i, i+j, p, q} \in G\left(V_{\text {odd }}\right)$, as $y$ runs through $F$, defined as follows.

For ease of notation we replace $g_{2 k+1, y}^{i, i+j, q}$ by $g ; s_{i}$ by $s ; e_{1}^{i, p}, \ldots, e_{2 s+1}^{i, p}$ by $e_{1}, \ldots, e_{2 s+1} ; s_{i+j}$ by $d$; and $e_{1}^{i+j, q}, \ldots, e_{2 d+1}^{i+j, q}$ by $f_{1}, \ldots, f_{2 d+1}$. If $v \in V_{\text {odd }}$ then $g$ fixes all basis vectors of (5) not listed below and

$$
\begin{gathered}
g\left(e_{2 k+1}\right)=e_{2 k+1}+y f_{1}, \quad g\left(f_{2}\right)=f_{2}-y e_{2 k+2}, \\
g\left(e_{2 k+3}\right)=e_{2 k+3}+y f_{3}, \quad g\left(f_{4}\right)=f_{4}-y e_{2 k+4}, \\
\vdots \\
g\left(e_{2(k+d)-1}\right)=e_{2(k+d)-1}+y f_{2 d-1}, \quad g\left(f_{2 d}\right)=f_{2 d}-y e_{2(k+d)}, \\
g\left(e_{2(k+d)+1}\right)=e_{2(k+d)+1}+y f_{2 d+1} .
\end{gathered}
$$

To see that $g$ indeed belongs to $G\left(V_{\text {odd }}\right)$ it suffices to verify that the matrices of $\varphi$ relative to the bases $\mathcal{B}$ and $\varphi(\mathcal{B})$ are equal. This is a simple computation involving basis vectors from at most two Gabriel blocks, and we omit it. 
3.18 Definition For $1 \leq i \leq t, 1 \leq p \leq m_{i}$ consider the 1-parameter subgroup of $G\left(V_{i}^{p}\right)$ formed by all $g_{x}^{i, p} \in G\left(V_{\text {odd }}\right)$, as $x$ runs through $F^{*}$, defined as follows.

For ease of notation we replace $g_{x}^{i, p}$ by $g ; s_{i}$ by $s$; and $e_{1}^{i, p}, e_{2}^{i, p}, \ldots, e_{2 s+1}^{i, p}$ by $e_{1}, e_{2}, \ldots, e_{2 s+1}$. If $v \in V_{i}^{p}$ then $g$ fixes all basis vectors of (5) not listed below and

$$
\begin{gathered}
g\left(e_{1}\right)=x e_{1}, g\left(e_{2}\right)=x^{-1} e_{2}, \ldots \\
g\left(e_{2 s-1}\right)=x e_{2 s-1}, g\left(e_{2 s}\right)=x^{-1} e_{2 s}, g\left(e_{2 s+1}\right)=x e_{2 s+1} .
\end{gathered}
$$

In this this case one easily verifies that $g \in G\left(V_{\text {odd }}\right)$.

3.19 Lemma Suppose $W$ is an $F$-vector space with a basis

$$
f_{1}^{1}, \ldots, f_{1}^{m}, f_{2}^{1}, \ldots, f_{2}^{m}, \ldots, f_{s}^{1}, \ldots, f_{s}^{m}
$$

For each $1 \leq p \leq m$ let

$$
E^{p}=f_{1}^{p}+f_{2}^{p}+\cdots+f_{s}^{p}
$$

Suppose $g \in \operatorname{End}_{F}(W)$ preserves each of the $m$-dimensional subspaces $\left(f_{k}^{1}, \ldots, f_{k}^{m}\right)$, where $1 \leq k \leq s$, and also the $m$-dimensional subspace $\left(E^{1}, \ldots, E^{m}\right)$. Suppose further that $g$ fixes all vectors $f_{1}^{1}, \ldots, f_{1}^{m}$. Then $g=1$.

Proof. From the invariance of the subspaces $\left(f_{k}^{1}, \ldots, f_{k}^{m}\right)$ we have

$$
g\left(f_{k}^{p}\right)=\sum_{1 \leq q \leq m} a_{k}^{p, q} f_{k}^{q}
$$

where $a_{k}^{p, q} \in F$. Since $g$ fixes $f_{1}^{1}, \ldots, f_{1}^{m}$

$$
a_{1}^{p, q}=\delta_{p, q}
$$

As $g$ is linear

$$
g\left(E^{p}\right)=\sum_{1 \leq k \leq s} \sum_{1 \leq q \leq m} a_{k}^{p, q} f_{k}^{q}=\sum_{1 \leq q \leq m} \sum_{1 \leq k \leq s} a_{k}^{p, q} f_{k}^{q} .
$$

But by the invariance of $\left(E^{1}, \ldots, E^{m}\right)$ we also have

$$
g\left(E^{p}\right)=\sum_{1 \leq q \leq m} b^{p, q} E^{q}=\sum_{1 \leq q \leq m} \sum_{1 \leq k \leq s} b^{p, q} f_{k}^{q},
$$


where $b^{p, q} \in F$. Therefore $a_{k}^{p, q}$ is independent of $k$, and in particular

$$
a_{k}^{p, q}=a_{1}^{p, q}=\delta_{p, q}
$$

as required.

\section{The split extension $1 \rightarrow N \rightarrow G \rightarrow G / N \rightarrow 1$}

4.1 Definition For $j \geq 1$ consider the subgroup $N_{j}$ of $G$ defined by

$$
N_{j}=\bigcap_{1 \leq i \leq t} G[V(i) / V(i+j)]
$$

and set $N=N_{1}$. Each $N_{j}$ is normal due to Lemma 3.13.

Note that

$$
N=N_{1} \supseteq N_{2} \supseteq \cdots \supseteq N_{t}=G\left[V_{\infty}\right], \quad N_{j}=G\left[V_{\infty}\right], \text { if } j \geq t .
$$

4.2 Definition For $1 \leq i \leq t$ let $E_{i}$ be the subgroup of $G\left(V_{i}\right)$ generated by all $g_{1, y}^{i, i, p, q}$ and all $g_{x}^{i, p}$. Let $E$ be the subgroup of $G\left(V_{\text {odd }}\right)$ generated by all $E_{i}, 1 \leq i \leq t$. Let

$$
E_{i}^{\prime}=G\left(V_{i}\right) \cap G\left\{V_{i}^{\dagger}\right\}, \quad 1 \leq i \leq t,
$$

and consider the internal direct product

$$
E^{\prime}=\prod_{1 \leq i \leq t} E_{i}^{\prime}
$$

4.3 Definition For $1 \leq i \leq t$ and $0 \leq k \leq s_{i}$ consider the $F G$-submodule $S_{2 k+1}^{i}$ of $V(i) / V(i+1)$, defined by

$$
S_{2 k+1}^{i}=\left(L^{2 k+1}(V) \cap R^{2\left(s_{i}-k\right)+1}(V) \cap V(i)+V(i+1)\right) / V(i+1) .
$$

We know from Lemma 3.10 that

$$
S_{2 k+1}^{i}=\left(\left(e_{2 k+1}^{i, 1}, \ldots, e_{2 k+1}^{i, m_{i}}\right) \oplus V(i+1)\right) / V(i+1)
$$

This yields the following decomposition of $F G$-modules

$$
V(i) / V(i+1)=\underset{0 \leq k \leq s_{i}}{\bigoplus} S_{2 k+1}^{i}
$$


4.4 Theorem The canonical map

$$
G \rightarrow \prod_{1 \leq i \leq t} \mathrm{GL}\left(S_{1}^{i}\right) \cong \prod_{1 \leq i \leq t} \mathrm{GL}_{m_{i}}(F)
$$

is a split group epimorphism with kernel $N$. Moreover,

$$
\begin{gathered}
E_{i}^{\prime}=E_{i} \cong \mathrm{GL}_{m_{i}}(F) \text { for all } 1 \leq i \leq t, \\
E^{\prime}=E
\end{gathered}
$$

and

$$
G=N \rtimes E .
$$

Proof. The above description of $S_{1}^{i}$ combined with Definitions 3.17 and 3.18 yield that each map

$$
E_{i} \rightarrow \mathrm{GL}\left(S_{1}^{i}\right), \quad 1 \leq i \leq t
$$

is surjective, whence the map (7) is also surjective.

To see that the kernel of (7) is $N$ we apply Lemmas 3.10, 3.16 and 3.19. Indeed, if $g \in G$ is in the kernel of $(7)$ then the $G$-invariance of $\operatorname{Rad}\left(\varphi-\varphi^{\prime}\right) \cap V(i)+V(i+1) / V(i+1)$ and all $S_{2 k+1}^{i}, 0 \leq k \leq s_{i}$, along with the fact that $g$ acts trivially on $S_{1}^{i}$, imply that $g$ acts trivially on $V(i) / V(i+1)$ for all $1 \leq i \leq t$, as required.

It follows that $G=N E$. But by definition $E \subseteq E^{\prime}$ and $E^{\prime} \cap N=1$. Therefore $E^{\prime}=E$, $G=N \rtimes E$, and $E_{i} \cong \mathrm{GL}_{m_{i}}(F)$ for all $1 \leq i \leq t$. Since it is obvious that $E$ is the internal direct product of the $E_{i} \subseteq E_{i}^{\prime}$, the proof is complete.

\section{Irreducible constituents of $V$ as an $F G$-module}

The series

$$
0 \subseteq V_{\infty} \subseteq{ }^{\infty} V \subseteq V
$$

reduces the search of irreducible constituents of the $F G$-module $V$ to that of the factors

$$
V_{\infty}, \quad{ }^{\infty} V / V_{\infty}, \quad V /{ }^{\infty} V
$$

First we consider the factor $V_{\infty}$. 
5.1 Theorem Each factor $V(i) / V(i+1), 1 \leq i \leq t$, of the series of $F G$-modules

$$
V_{\infty}=V(1) \supset V(2) \supset \cdots \supset V(t) \supset V(t+1)=0
$$

is equal to the direct sum of $s_{i}+1$ isomorphic irreducible $F G$-modules of dimension $m_{i}$

$$
V(i) / V(i+1)=\underset{0 \leq k \leq s_{i}}{\bigoplus} S_{2 k+1}^{i}
$$

Moreover,

$$
G\left[S_{2 k+1}^{i}\right]=N \rtimes \prod_{l \neq i} E_{i}
$$

and as a module for

$$
G / G\left[S_{2 k+1}^{i}\right] \cong E_{i} \cong \mathrm{GL}_{m_{i}}(F),
$$

$S_{2 k+1}^{i}$ is isomorphic to the natural $m_{i}$-dimensional module over $F$, namely $F^{m_{i}}$.

Proof. This is clear from section $\S 4$.

Next we make preliminary remarks about the factor ${ }^{\infty} V / V_{\infty}$.

5.2 Definition Let $V^{\infty}=L^{\infty}(V) \cap R^{\infty}(V)$ and ${ }_{\infty} V=L_{\infty}(V)+R_{\infty}(V)$.

By construction these are $F G$-submodules of $V$, and we know from [3] that

$$
{ }_{\infty} V=V_{\text {even }} \perp V_{\infty} \text { and } V^{\infty}=V_{\text {ndeg }} \perp V_{\infty}
$$

Observe that ${ }^{\infty} V / V_{\infty}$ is a bilinear space, with even and non-degenerate parts equal to

$$
{ }_{\infty} V / V_{\infty} \cong V_{\text {even }} \text { and } V^{\infty} / V_{\infty} \cong V_{\text {ndeg }}
$$

Since the odd part of ${ }^{\infty} V / V_{\infty}$ is equal to zero, we know from [3] that even and nondegenerate parts of ${ }^{\infty} V / V_{\infty}$ are unique, so

$$
G\left({ }^{\infty} V / V_{\infty}\right)=G\left({ }_{\infty} V / V_{\infty}\right) \times G\left(V^{\infty} / V_{\infty}\right)
$$

It follows that the canonical map

$$
G(V) \rightarrow G\left({ }^{\infty} V / V_{\infty}\right)
$$


is a group epimorphism whose restriction to $G\left(V_{\text {even }}\right) \times G\left(V_{\text {ndeg }}\right)$ is an isomorphism. Since the kernel of this map is $G\left[{ }^{\infty} V / V_{\infty}\right]$, whose intersection with $G\left(V_{\text {even }}\right) \times G\left(V_{\text {ndeg }}\right)$ is trivial, we obtain the decomposition

$$
G(V)=G\left[{ }^{\infty} V / V_{\infty}\right] \rtimes G\left(V_{\text {even }}\right) \times G\left(V_{\text {ndeg }}\right) .
$$

It follows from the above considerations that the study of the $F G$-module ${ }^{\infty} V / V_{\infty}$ reduces to the study of the $F G\left(V_{\text {even }}\right)$-module $V_{\text {even }}$ and the $F G\left(V_{\text {ndeg }}\right)$-module $V_{\text {ndeg }}$.

\section{The split extension $1 \rightarrow G\left[V_{\infty}\right] \rightarrow N \rightarrow N / G\left[V_{\infty}\right] \rightarrow 1$}

The very definition of the groups $N_{j}$ gives

$$
\left[N_{i}, N_{j}\right] \subseteq N_{i+j}
$$

so $\left(N_{j}\right)_{1 \leq j \leq t}$ yields a $G$-invariant descending central series for $N / G\left[V_{\infty}\right]$. By abuse of language we shall sometimes say that $\left(N_{j}\right)_{1 \leq j \leq t}$ and like series are central series for $N / G\left[V_{\infty}\right]$.

6.1 Notation If $g_{1}, g_{2} \in G$ then $\left[g_{2}, g_{1}\right]=g_{2}^{-1} g_{1}^{-1} g_{2} g_{1}$. If $n>2$ and $g_{1}, \ldots, g_{n-1}, g_{n} \in G$ then $\left[g_{n}, g_{n-1}, \ldots, g_{1}\right]=\left[g_{n},\left[g_{n-1}, \ldots, g_{1}\right]\right]$.

6.2 Theorem The nilpotency class of $N / G\left[V_{\infty}\right]$ is $t-1$.

Proof. By the above comments the nilpotency class of $N / G\left[V_{\infty}\right]$ is at most $t-1$. If $t>1$ then

$$
\left[g_{1,1}^{t-1, t, 1,1}, \ldots, g_{1,1}^{2,3,1,1}, g_{1,1}^{1,2,1,1}\right] \neq 1
$$

so the result follows.

The series $\left(N_{j}\right)_{1 \leq j \leq t}$ needs to be refined in order to obtain sharper results on the structure of $N / G\left[V_{\infty}\right]$.

\subsection{Generators for nilpotent group $N / G\left[V_{\infty}\right]$}

6.3 Definition For $k \geq 0$ define the normal subgroup $M_{2 k+1}$ of $G$ by

$$
M_{2 k+1}=G\left[L^{2 k+1}(V) \cap V_{\infty}\right] \cap N .
$$


We further define $M_{-1}=N$.

Note that

$$
N=M_{-1} \supseteq M_{1} \supseteq M_{3} \supseteq \cdots \supseteq M_{2 s_{1}+1}=G\left[V_{\infty}\right],
$$

with

$$
M_{2 k+1}=G\left[V_{\infty}\right], \quad k \geq s_{1} .
$$

We use the $G$-invariant series $\left(M_{2 k-1}\right)_{0 \leq k}$ to refine the $G$-invariant decreasing central series $\left(N_{j}\right)_{1 \leq j}$ for $N / G\left[V_{\infty}\right]$, obtaining the $G$-invariant decreasing central series for $N / G\left[V_{\infty}\right]$

$$
N_{j, 2 k-1}=\left(N_{j} \cap M_{2 k-1}\right) N_{j+1}, \quad 0 \leq k, 1 \leq j .
$$

We have $N_{j, 2 s_{1}+1}=N_{j+1}=N_{j+1,-1}$ and

$$
\begin{gathered}
N_{1}=N_{1,-1} \supseteq N_{1,1} \supseteq N_{1,3} \supseteq \cdots \supseteq N_{1,2 s_{1}-1} \supseteq N_{2} \supseteq \cdots \\
N_{t-1}=N_{t-1,-1} \supseteq N_{t-1,1} \supseteq \cdots \supseteq N_{t-1,2 s_{1}-1} \supseteq N_{t-1,2 s_{1}+1}=N_{t}=1 .
\end{gathered}
$$

6.4 Theorem Let $k \geq 0$. Then

$$
M_{2 k-1} \subseteq G\left[L^{2 k+1}(V) \cap V_{\infty} / L(V) \cap V_{\infty}\right]
$$

Proof. We may assume $k \geq 1$, for otherwise the result is trivial. Since

$$
L^{2 k+1}(V) \cap V_{\infty}=L^{2 k+1}(V) \cap V_{1} \oplus \cdots \oplus L^{2 k+1}(V) \cap V_{t},
$$

it suffices to show

$$
(g-1) L^{2 k+1}(V) \cap V_{i} \subseteq L(V) \cap V_{\infty}, \quad g \in M_{2 k-1}, 1 \leq i \leq t .
$$

Fix $i, 1 \leq i \leq t$. If $k>s_{i}$ then $L^{2 k+1}(V) \cap V_{i}=L^{2 k-1}(V) \cap V_{i}$, so (11) holds. Suppose $k \leq s_{i}$. Then

$$
L^{2 k+1}(V) \cap V_{i}=L^{2 k-1}(V) \cap V_{i} \oplus L^{2 k+1}(V) \cap R^{2\left(s_{i}-k\right)+1}(V) \cap V_{i},
$$

so (11) is equivalent to

$$
(g-1) L^{2 k+1}(V) \cap R^{2\left(s_{i}-k\right)+1}(V) \cap V_{i} \subseteq L(V) \cap V_{\infty}, \quad g \in M_{2 k-1} .
$$


By Lemma 3.10 a basis for $L^{2 k+1}(V) \cap R^{2\left(s_{i}-k\right)+1}(V) \cap V_{i}$ is given by $\left(e_{2 k+1}^{i, p}\right)_{1 \leq p \leq m_{i}}$. Let $g \in M_{2 k-1}$ and fix $p, 1 \leq p \leq m_{i}$. We are reduced to show that $g$ fixes $e_{2 k+1}^{i, p}$ modulo $L(V) \cap V_{\infty}$. Since $g \in N$, we have

$$
g\left(e_{2 k+1}^{i, p}\right)=e_{2 k+1}^{i, p}+z
$$

where $z \in L^{2 k+1}(V) \cap V(i+1)$. Suppose $z \notin L(V) \cap V(i+1)$. Then

$$
\left\langle g e_{2 k+1}^{i, p}, e_{2 c}^{l, q}\right\rangle \neq 0
$$

for some $t \geq l \geq i+1,1 \leq q \leq m_{l}$ and $1 \leq c \leq s_{l}$. Then

$$
\left\langle e_{2 k+1}^{i, p}, g^{-1} e_{2 c}^{l, q}\right\rangle \neq 0
$$

so $g^{-1} e_{2 c}^{l, q}$ has non-zero coefficient in $e_{2 k}^{i, p}$. But $g \in M_{2 k-1}$ and $l>i$, so

$$
0 \neq\left\langle g^{-1} e_{2 c}^{l, q}, e_{2 k-1}^{i, p}\right\rangle=\left\langle e_{2 c}^{l, q}, g e_{2 k-1}^{i, p}\right\rangle=\left\langle e_{2 c}^{l, q}, e_{2 k-1}^{i, q}\right\rangle=0
$$

a contradiction.

6.5 Definition Let $1 \leq j$ and $0 \leq k$. Set

$$
I(j, k)=\left\{i \geq 1 \mid 1 \leq i \leq t-j \text { and } k \leq s_{i}-s_{i+j}\right\}
$$

Note that $I(j, k)=\emptyset$ if $j \geq t$ or $k>s_{1}$. For $i \geq 1$ we set $X(i, j, 2 k-1)=\emptyset$ of $i \notin I(j, k)$ and otherwise

$$
X(i, j, 2 k-1)=\left\{g_{2 k+1, y}^{i, i+j, p} \mid 1 \leq p \leq m_{i}, 1 \leq q \leq m_{i+j}, y \in F\right\}
$$

We further define

$$
X(j, 2 k-1)=\cup_{i \geq 1} X(i, j, 2 k-1), X(j)=\cup_{k \geq 0} X(j, 2 k-1), X=\cup_{j \geq 1} X(j) .
$$

6.6 Theorem Let $k \geq 0$ and $j \geq 1$. Then $X(j, 2 k-1) \subseteq M_{2 k-1} \cap N_{j}$ and the quotient group

$$
\left(M_{2 k-1} \cap N_{j}\right) M_{2 k+1} /\left(M_{2 k-1} \cap N_{j+1}\right) M_{2 k+1}
$$

is generated by the classes of all elements in $X(j, 2 k-1)$. In particular, this quotient is trivial if $I(j, k)=\emptyset$ (the converse is true and proved in Theorem 6.19 below). 
Proof. Clearly $X(j, 2 k-1) \subseteq M_{2 k-1} \cap N_{j}$. Let $g \in M_{2 k-1} \cap N_{j}$. We claim that for each $i, 1 \leq i \leq t$, there exists

$$
h_{i} \in<X(i, j, 2 k-1)><\underset{r>j}{\cup} X(i, r, 2 k-1)>
$$

such that $h_{i} g$ is the identity on $L^{2 k+1}(V) \cap V_{i}$.

To prove our claim we fix $i, 1 \leq i \leq t$. If $k>s_{i}$ we take $h_{i}=1$. Suppose $k \leq s_{i}$. In view of $g \in M_{2 k-1}$ and the decomposition (12), it suffices to choose $h_{i}$ as in (14), so that $h_{i} g$ fixes every basis vector $e_{2 k+1}^{i, p}, 1 \leq p \leq m_{i}$, of $L^{2 k+1}(V) \cap R^{2\left(s_{i}-k\right)+1}(V) \cap V_{i}$. By Theorem 6.4 and the fact that $g \in N_{j}$, for each $1 \leq p \leq m_{i}$ we have

$$
g\left(e_{2 k+1}^{i, p}\right)=e_{2 k+1}^{i, p}+z
$$

where $z \in L(V) \cap R^{2\left(s_{i}-k\right)+1}(V) \cap V(i+j)$. If $i+j>t$ then $z=0$ and if $i+j \leq t$ but $k>s_{i}-s_{i+j}$ then $z=0$ as well by Lemma 3.9. In both cases we take $h_{i}=1$. Otherwise, again by Lemma 3.9, we may write

$$
z=z_{i+j}+\cdots+z_{i+j+l}
$$

where $l \geq 0, z_{i+j+b} \in L(V) \cap R^{2\left(s_{i}-k\right)+1}(V) \cap V_{i+j+b}$ and $k \leq s_{i}-s_{i+j+b}$ for all $0 \leq b \leq l$.

It is know clear from the very definition of the $g_{2 k+1, y}^{i, i+j+b, p, q}$ that we may choose $h_{i}$ as in (14) so that $h_{i} g$ is the identity on each $e_{2 k+1}^{i, p}$.

By construction $h_{i}$ is the identity on $\underset{l \neq i}{\oplus} V_{l}$. Therefore our claim and the decomposition (10) imply that $h_{1} \cdots h_{t} g$ is the identity on $L^{2 k+1}(V) \cap V_{\infty}$, i.e. $h_{1} \cdots h_{t} g \in M_{2 k+1}$. But if $j<r$ then $X(i, r, 2 k-1) \subseteq M_{2 k-1} \cap N_{j+1}$, so the class of $g$ is equal to the product of the classes of elements from $X(j, 2 k-1)$, as required.

6.7 Theorem Let $k \geq 0$ and $j \geq 1$. Then $X(j, 2 k-1) \subseteq M_{2 k-1} \cap N_{j}$ and the quotient group

$$
\left(M_{2 k-1} \cap N_{j}\right) N_{j+1} /\left(M_{2 k+1} \cap N_{j}\right) N_{j+1}
$$

is generated by the classes of all elements in $X(j, 2 k-1)$. In particular, this quotient is trivial if $I(j, k)=\emptyset$ (the converse is true and proved in Theorem 6.19 below). 
Proof. This follows from Theorem 6.6 and the Butterfly Lemma.

6.8 Theorem Let $1 \leq j<j^{\prime} \leq t$. Then $X(l) \subseteq N_{j}$ for all $j \leq l<j^{\prime}$ and $N_{j} / N_{j^{\prime}}$ is generated the classes of all these elements. In particular $N / G\left[V_{\infty}\right]$ is generated by the classes of all elements in $X$.

Proof. This follows from Theorem 6.7 via the series $N_{j, 2 k-1}$.

6.9 Definition Let $U$ be the subgroup of $G\left(V_{\text {odd }}\right) \cap N$ generated by $X$ and let

$$
U^{\prime}=N \cap G\left(V_{\text {odd }}\right) \cap G\left[V_{1}^{\dagger}\right] \cap G\left[V_{1}^{\dagger} \oplus V_{2}^{\dagger} / V_{1}^{\dagger}\right] \cap \cdots \cap G\left[V_{1}^{\dagger} \oplus \cdots \oplus V_{t}^{\dagger} / V_{1}^{\dagger} \oplus \cdots \oplus V_{t-1}^{\dagger}\right] .
$$

It is clear that $N / G\left[V_{\infty}\right]$ is a unipotent subgroup of $V_{\infty}$. The next result shows that $N=G\left[V_{\infty}\right] \rtimes U$, where $U$ is unipotent in $V$.

\subsection{Theorem $\quad U^{\prime}=U$ is unipotent and $N=G\left[V_{\infty}\right] \rtimes U$.}

Proof. From Theorem 6.8 we infer $N=G\left[V_{\infty}\right] U$. The definition of $X$ yields $U \subseteq U^{\prime}$. But by Lemma 3.1

$$
G\left[V_{\infty}\right] \cap U^{\prime} \subseteq G\left[V_{\infty}\right] \cap G\left[V /{ }^{\infty} V\right] \cap U^{\prime}=1
$$

It follows that $U=U^{\prime}$ and $N=G\left[V_{\infty}\right] \rtimes U$.

Finally, if $g \in U$ then $g-\left.1\right|_{V_{\text {odd }}^{\dagger}}$ is nilpotent by the very definition of $U^{\prime}$, and $g-\left.1\right|_{\infty}$ is nilpotent since $g \in N$. Thus $g$ is unipotent.

\subsection{Irreducible constituents of the $F G$-module $V /{ }^{\infty} V$}

We have accumulated enough information to determine the irreducible constituents of the $F G$-module $V /{ }^{\infty} V$.

6.11 Definition Let $\mathbf{t}=t$ if $s_{t}>0$ (i.e. $\operatorname{Rad}(V)=0$ ) and $\mathbf{t}=t-1$ if $s_{t}=0$ (i.e. $\operatorname{Rad}(V) \neq 0)$.

6.12 Definition For $1 \leq i \leq \mathbf{t}$ let

$$
(i) V=V_{1}^{\dagger} \oplus \cdots \oplus V_{i}^{\dagger} \oplus^{\infty} V
$$


and set

$$
\text { (0) } V={ }^{\infty} V \text {. }
$$

6.13 Lemma If $0 \leq i \leq \mathbf{t}$ then $(i) V$ is an $F G$-submodule of $V$.

Proof. We may assume that $i \geq 1$. From the identity $N=G\left[V_{\infty}\right] \rtimes U$, the inclusion $G\left[V_{\infty}\right] \subseteq G\left[V /{ }^{\infty} V\right]$ of Lemma 3.1, and the characterization of $U$ given in Theorem 6.10 we infer that $(i) V$ is preserved by $N$. The very definition of $E$ and the identity $G=N \rtimes E$ of Theorem 4.4 allow us to conclude that $(i) V$ is in fact $G$-invariant.

6.14 Lemma If $1 \leq i \leq \mathbf{t}$ then $(i) V /(i-1) V$ is an $F G$-module acted upon trivially by $N$.

Proof. The characterization of of $U$ given in Theorem 6.10 shows that $U$ acts trivially on $(i) V /(i-1) V$, while Lemma 3.1 shows that $G\left[V_{\infty}\right]$ also acts trivially on $(i) V /(i-1) V$. Since $N=G\left[V_{\infty}\right] \rtimes U$, the result follows.

6.15 Theorem Each factor $(i) V / V(i-1), 1 \leq i \leq \mathbf{t}$, of the series of $F G$-modules

$$
{ }^{\infty} V=(0) V \subset(1) V \subset \cdots \subset(\mathbf{t}-1) V \subset(\mathbf{t}) V=V
$$

is isomorphic to the direct sum of $s_{i}$ isomorphic irreducible $F G$-modules of dimension $m_{i}$, namely

$$
Q_{2 k}^{i}=\left(\left(e_{2 k}^{i, 1}, \ldots, e_{2 k}^{i, m_{i}}\right) \oplus(i-1) V\right) /(i-1) V
$$

where $1 \leq k \leq s_{i}$. Moreover,

$$
G\left[Q_{2 k}^{i}\right]=N \rtimes \prod_{l \neq i} E_{i}
$$

and as a module for

$$
G / G\left[Q_{2 k}^{i}\right] \cong E_{i} \cong \mathrm{GL}_{m_{i}}(F)
$$

$Q_{2 k}^{i}$ is isomorphic to the natural $m_{i}$-dimensional module over $F$, namely $F^{m_{i}}$.

Proof. This follows from Lemma 6.14 and (8). 


\subsection{A refined $G$-invariant descending central series for $N / G\left[V_{\infty}\right]$}

In this section we construct a $G$-invariant descending central series for $N / G\left[V_{\infty}\right]$ each of whose factors is naturally an irreducible $F G$-module, whose isomorphism type we explicitly determine, all of which are connected to the irreducible constituents of the $F G$-module $V_{\infty}$, as described in Theorem 5.1

6.16 Theorem For each $j \geq 1$ there is a canonical group embedding

$$
N_{j} / N_{j+1} \rightarrow \underset{1 \leq i \leq t-1}{\oplus} \operatorname{Hom}_{F}(V(i) / V(i+1), V(i+j) / V(i+j+1)),
$$

whose image is an $F$-vector subspace of the codomain. By transferring this $F$-vector space structure to $N_{j} / N_{j+1}$ the above map becomes an embedding of $F G$-modules.

Proof. Recall first of all that since $V(i) / V(i+1)$ and $V(i+j) / V(i+j+1)$ are $F G$-modules, so is $\operatorname{Hom}_{F}(V(i) / V(i+1), V(i+j) / V(i+j+1))$ in a natural manner.

Define the map $N_{j} / N_{j+1} \rightarrow \operatorname{Hom}_{F}(V(i) / V(i+1), V(i+j) / V(i+j+1)), 1 \leq i \leq t-1$, by $[g] \mapsto g_{i}$, where $g \in N_{j}$ and

$$
g_{i}(v+V(i+1))=(g-1)(v)+V(i+j+1), \quad v \in V(i) .
$$

Then let $N_{j} / N_{j+1} \rightarrow \underset{1 \leq i \leq t-1}{\oplus} \operatorname{Hom}_{F}(V(i) / V(i+1), V(i+j) / V(i+j+1))$ be defined by

$$
[g] \mapsto\left(g_{1}, \ldots, g_{t-1}\right), \quad g \in N_{j}
$$

The definitions of all objects involved and the identity

$$
g h-1=(g-1)(h-1)+(h-1)+(g-1), \quad g, h \in G
$$

show that our map is a well-defined group monomorphism which is compatible with the action of $G$ on both sides.

It remains to show that the image of our map is an $F$-subspace of the codomain. By Theorem $6.8 N_{j} / N_{j+1}$ is generated by all $g N_{j+1}$ as $g$ runs through $X(j)$. Since our map is a group homomorphism, it suffices to show that $k(g-1)+1 \in N_{j}$ for all $g \in X(j)$ and $k \in F$. But Definition 3.17 makes this clear, so the proof is complete. 
6.17 Note Let $k \geq 0$ and $j \geq 1$. Notice that the group $N_{j, 2 k-1} / N_{j, 2 k+1}$ is a section of the $F G$-module $N_{j} / N_{j+1}$ of Theorem 6.16 , and as such inherits a natural structure of FG-module.

6.18 Definition Let $k \geq 0$ and $j \geq 1$. For each $1 \leq i \leq t$ define $N_{i, j, 2 k-1}=\left\{g \in N_{j, 2 k-1} \mid(g-1) L^{2 k+1}(V) \cap V(l) \subseteq V(l+j+1)\right.$ for all $\left.1 \leq l \leq t, l \neq i\right\}$.

Note that for each $1 \leq i \leq t, N_{i, j, 2 k-1}$ is a normal subgroup of $G$ containing $N_{j, 2 k+1}$. As a section of $N_{j} / N_{j+1}$, the the group $N_{i, j, 2 k-1} / N_{j, 2 k+1}$ is also an $F G$-module.

Recall at this point the meaning of the $F G$-modules $S_{2 k+1}^{i}$, as given in Definition 4.3.

6.19 Theorem Let $k \geq 0$ and $j \geq 1$. For each $i \in I(j, k)$ there is a canonical isomorphism of $F G$-modules

$$
Y_{i}: N_{i, j, 2 k-1} / N_{j, 2 k+1} \rightarrow \operatorname{Hom}_{F}\left(S_{2 k+1}^{i}, S_{1}^{i+j}\right)
$$

Moreover, we have $X(i, j, 2 k-1) \subseteq N_{i, j, 2 k-1}$, and $N_{i, j, 2 k-1} / N_{j, 2 k+1}$ is generated by the classes of all elements in $X(i, j, 2 k-1)$.

There is a canonical isomorphism of $F G$-modules

$$
Y:\left(N_{j} \cap M_{2 k-1}\right) N_{j+1} /\left(N_{j} \cap M_{2 k+1}\right) N_{j+1} \rightarrow \underset{i \in I(j, k)}{\oplus} \operatorname{Hom}_{F}\left(S_{2 k+1}^{i}, S_{1}^{i+j}\right) .
$$

induced by the $Y_{i}$. The dimension of both of these modules, say $d_{j, 2 k-1}$, is equal to $d_{j, 2 k-1}=\sum_{i \in I(j, k)} m_{i} m_{i+j}$. Moreover, we have

$$
\left(N_{j} \cap M_{2 k-1}\right) N_{j+1} /\left(N_{j} \cap M_{2 k+1}\right) N_{j+1}=\prod_{i \in I(j, k)} N_{i, j, 2 k-1} / N_{j, 2 k+1} .
$$

Proof. Let $i \in I(j, k)$. In the spirit of Theorem 6.16 we consider the map

$$
Y_{i}:\left(N_{j} \cap M_{2 k-1}\right) N_{j+1} /\left(N_{j} \cap M_{2 k+1}\right) N_{j+1} \rightarrow \operatorname{Hom}_{F}\left(S_{2 k+1}^{i}, S_{1}^{i+j}\right)
$$

given by $[g] \mapsto g_{i}$, where $g \in\left(N_{j} \cap M_{2 k-1}\right) N_{j+1}$ and

$$
g_{i}(v+V(i+1))=(g-1)(v)+V(i+j+1),
$$


for all $v \in L^{2 k+1}(V) \cap R^{2\left(s_{i}-k\right)+1}(V) \cap V(i)+V(i+1)$.

STEP I: $Y_{i}$ is a well-defined homomorphism of $F G$-modules.

Let $g \in\left(N_{j} \cap M_{2 k-1}\right) N_{j+1}$. We claim that $(g-1) v \in L(V) \cap V(i+j)+V(i+j+1)$ for all $v \in L^{2 k+1}(V) \cap R^{2\left(s_{i}-k\right)+1}(V) \cap V(i)+V(i+1)$, and that $(g-1) v+V(i+j+1)$ depends only on $v+V(i+j)$, that is, $g_{i}$ is a well-defined function $S_{2 k+1}^{i} \rightarrow S_{1}^{i+j}$.

Since $N_{j} \cap M_{2 k-1}$ and $N_{j+1}$ are normal subgroups of $G$ we may write $g=g_{1} g_{2}$, where $g_{1} \in N_{j} \cap M_{2 k-1}$ and $g_{2} \in N_{j+1}$. Then

$$
g-1=g_{1} g_{2}-1=g_{1}\left(g_{2}-1+1\right)-1=g_{1}\left(g_{2}-1\right)+\left(g_{1}-1\right) .
$$

Suppose first $v \in V(i+1)$. Then from $g \in N_{j}$ it follows $(g-1) v \in V(i+j+1)$. Suppose next $v \in L^{2 k+1}(V) \cap R^{2\left(s_{i}-k\right)+1}(V) \cap V(i)$. Then $g_{2} \in N_{j+1}$ implies $\left(g_{2}-1\right) v \in V(i+j+1)$, while Theorem 6.4 and the definition of $N_{j}$ give $\left(g_{1}-1\right) v \in L(V) \cap R^{2\left(s_{i}-k\right)+1}(V) \cap V(i+j)$. But $i \in I(j, k)$, so $s_{i}-k \geq s_{i+j}$ and therefore Lemma 3.7 gives

$$
L(V) \cap R^{2\left(s_{i}-k\right)+1}(V) \cap V(i+j)+V(i+j+1)=L(V) \cap V(i+j)+V(i+j+1) .
$$

Thus $\left(g_{2}-1\right) v \in L(V) \cap V(i+j)+V(i+j+1)$. Our claim now follows from the above considerations.

We next claim that $g_{i}$ depends only on the the class $[g]=g\left(N_{j} \cap M_{2 k+1}\right) N_{j+1}$ of g. For this purpose let $h \in\left(N_{j} \cap M_{2 k+1}\right) N_{j+1}$. We may again write $h=h_{1} h_{2}$, where $h_{1} \in N_{j} \cap M_{2 k+1}$ and $h_{2} \in N_{j+1}$. Let $v \in L^{2 k+1}(V) \cap R^{2\left(s_{i}-k\right)+1}(V) \cap V(i)$. As above $\left(h_{2}-1\right) \in V(i+j+1)$, while the very definition of $M_{2 k+1}$ ensures that $\left(h_{1}-1\right) v=0$. It follows that $(h-1) v \in V(i+j+1)$, thereby proving our claim.

Since it is clear that $g_{i}$ is not just a function $S_{2 k+1}^{i} \rightarrow S_{1}^{i+j}$ but also a linear map, what we have proven so far is that $Y_{i}$ is a well-defined function.

We next claim that $Y_{i}$ is a group homomorphism. Indeed, let $g, h \in\left(N_{j} \cap M_{2 k-1}\right) N_{j+1}$. Let $v \in L^{2 k+1}(V) \cap R^{2\left(s_{i}-k\right)+1}(V) \cap V(i)$. Then

$$
(g h-1) v+V(i+j+1)=(h-1) v+(g-1) v+(g-1)(h-1) v+V(i+j+1) .
$$

Since $(h-1) v \in L(V) \cap V(i+j)+V(i+j+1) \cap V(i+j)$, and $g \in N_{j}$ gives $(g-1) V(i+j) \subseteq$ $V(i+j+1)$, we deduce $(g-1)(h-1) v \in V(i+j+1)$. It follows that

$$
(g h-1) v+V(i+j+1)=(h-1) v+(g-1) v+V(i+j+1),
$$


thereby proving our claim. We only remaining details to check is that $Y_{i}$ commutes with the actions of $G$ and $F$, but this is straightforward and we can safely omit the details.

STEP II: $Y_{i}$ restricted to a subgroup $R_{i}$ is an isomorphism.

We again let $i \in I(j, k)$. By Theorem 6.7 we know that $X(i, j, 2 k-1)$ is contained in $\left(N_{j} \cap M_{2 k-1}\right) N_{j+1}$. Let $R_{i}$ be the subgroup of $\left.\left(N_{j} \cap M_{2 k-1}\right) N_{j+1} / N_{j} \cap M_{2 k+1}\right) N_{j+1}$ generated by the classes of all elements in $X(i, j, 2 k-1)$. The construction of $R_{i}$ along with Definitions 3.17 and 6.18 show that

$$
R_{i} \subseteq N_{i, j, 2 k-1} / N_{j, 2 k+1}
$$

Moreover, the definition of $Y_{i}$ along with Definition 3.17 show that the images under $Y_{i}$ of the elements

$$
g_{2 k+1,1}^{i, i+j, p, q} N_{j, 2 k+1} \in R_{i}, \quad 1 \leq p \leq m_{i}, 1 \leq q \leq m_{i+j}
$$

form an $F$-basis of $\operatorname{Hom}_{F}\left(S_{2 k+1}^{i}, S_{1}^{i+j}\right)$. From Theorem 5.1 we know that this space is $m_{i} m_{i+j}$-dimensional. But the $m_{i} m_{i+j}$ elements (18) generate $R_{i}$ as a vector space. It follows that the restriction of $Y_{i}$ to $R_{i}$ is an isomorphism and

$$
\operatorname{dim} R_{i}=m_{i} m_{i+j}
$$

STEP III: $Y$ is an isomorphism.

Let

$$
Y: N_{j, 2 k-1} / N_{j, 2 k+1} \rightarrow \underset{i \in I(j, k)}{\oplus} \operatorname{Hom}_{F}\left(S_{2 k+1}^{i}, S_{1}^{i+j}\right)
$$

be the homomorphism of $F G$-modules induced by the $Y_{i}, i \in I(j, k)$. The very definitions of $Y_{i}$ and $N_{i, j, 2 k-1}$ show that

$$
N_{i, j, 2 k-1} / N_{j, 2 k+1} \subseteq \operatorname{ker} Y_{i^{\prime}}, \quad i \neq i^{\prime} \in I(j, k)
$$

We deduce from (17) that

$$
R_{i} \subseteq \operatorname{ker} Y_{i^{\prime}}, \quad i \neq i^{\prime} \in I(j, k) .
$$

It follows that the image under $Y$ of the product of the subgroups $R_{i}$ of $N_{j, 2 k-1} / N_{j, 2 k+1}$, as $i$ ranges through $I(j, k)$, is equal to $\underset{i \in I(j, k)}{\oplus} \operatorname{Hom}_{F}\left(S_{2 k+1}^{i}, S_{1}^{i+j}\right)$. Since each summand 
in this space has dimension $m_{i} m_{i+j}$, the entire space has dimension $d_{j, 2 k-1}$. But from Theorem 6.7 we see that

$$
\operatorname{dim} N_{j, 2 k-1} / N_{j, 2 k+1} \leq d_{j, 2 k-1}
$$

Since by above $Y$ is an epimorphism, we deduce that $Y$ is an isomorphism and

$$
\operatorname{dim} N_{j, 2 k-1} / N_{j, 2 k+1}=d_{j, 2 k-1}
$$

STEP IV: $N_{j, 2 k-1} / N_{j, 2 k+1}$ is internal direct product of the $R_{i}$.

By Theorem 6.7 we know that $N_{j, 2 k-1} / N_{j, 2 k+1}$ is generated as an $F$-vector space by its subspaces $R_{i}, i \in I(j, k)$. We infer from (19) and (21) that as a vector space $N_{j, 2 k-1} / N_{j, 2 k+1}$ is the direct sum of the $R_{i}$, therefore as groups we have the following internal direct product decomposition

$$
N_{j, 2 k-1} / N_{j, 2 k+1}=\prod_{i \in I(j, k)} R_{i}
$$

STEP V: $N_{i, j, 2 k-1} / N_{j, 2 k+1}=R_{i}$ for all $i \in I(j, k)$.

Let $i \in I(j, k)$. In view of (17) and (19) it suffices to prove that $\operatorname{dim} N_{i, j, 2 k-1} / N_{j, 2 k+1} \leq$ $m_{i} m_{i+j}$. For this purpose let $P_{i}$ denote the product of all $N_{i^{\prime}, j, 2 k-1} / N_{j, 2 k+1}, i \neq i^{\prime} \in I(j, k)$. From (20) we see that $P_{i}$ is contained in the kernel of $Y_{i}$. This fact and a new application of (20) yield that $P_{i} \cap\left(N_{i, j, 2 k-1} / N_{j, 2 k+1}\right)$ is contained in the kernel of $Y$. But $Y$ is an isomorphism, so

$$
P_{i} \cap\left(N_{i, j, 2 k-1} / N_{j, 2 k+1}\right)=1, \quad i \in I(j, k) .
$$

But $P_{i}$ contains all classes of elements in $X\left(i^{\prime}, j, 2 k-1\right), i \neq i^{\prime} \in I(j, k)$, so by Theorem 6.7 the dimension of the quotient space of $N_{j, 2 k-1} / N_{j, 2 k+1}$ by $P_{i}$ has dimension at most $m_{i} m_{i+j}$. This and (22) imply

$$
\operatorname{dim} N_{i, j, 2 k-1} / N_{j, 2 k+1} \leq m_{i} m_{i+j}
$$

as required. This completes the proof of the theorem.

6.20 Theorem $\operatorname{dim} U=\sum_{1 \leq i<j \leq t}\left(s_{i}-s_{j}+1\right) m_{i} m_{j}$. 
Proof. By Theorem 6.19 we have

$$
\operatorname{dim} U=\sum_{1 \leq j} \sum_{0 \leq k} \sum_{i \in I(j, k)} m_{i} m_{i+j}=\sum_{1 \leq i<j \leq t}\left(s_{i}-s_{j}+1\right) m_{i} m_{j} .
$$

6.21 Definition For each $j \geq 1$ let $k(j)$ be the largest integer $k$ such that $I(j, k)$ is non-empty.

6.22 Theorem There is a canonical $G$-invariant descending central series for $N / G\left[V_{\infty}\right]$ each of whose factors is naturally an irreducible $F G$-module, which can be obtained as follows.

We start with $G$-invariant decreasing central series

$$
\left(N_{j, 2 k-1} / G\left[V_{\infty}\right]\right)_{1 \leq j, 0 \leq k}
$$

of $N / G\left[V_{\infty}\right]$ defined in (9) and refine it by means of the decomposition (16). The only non-trivial factors thus arising are

$$
N_{i, j, 2 k-1} / N_{j, 2 k-1} \cong_{F G} \operatorname{Hom}_{F}\left(S_{2 k+1}^{i}, S_{1}^{i+j}\right),
$$

where $1 \leq j<t-1,0 \leq k \leq k(j), i \in I(j, k)$, the dimension of $\operatorname{Hom}_{F}\left(S_{2 k+1}^{i}, S_{1}^{i+j}\right)$ is $m_{i} m_{i+j}$, and the $S_{2 k+1}^{i}, S_{1}^{i+j}$ are amongst the irreducible constituents of the $F G$-module $V_{\infty}$ determined in Theorem 5.1.

Each of the factors (23) is an irreducible $F G$-module whose isomorphism type depends only on $i$ and $j$, and whose multiplicity in the series is exactly $s_{i}-s_{j}+1$. Moreover, $G\left[N_{i, j, 2 k-1} / N_{j, 2 k-1}\right]$ contains $N$ and all $E_{l}$, where $1 \leq l \leq t, l \neq i, i+j$, and as a module over

$$
G / G\left[N_{i, j, 2 k-1} / N_{j, 2 k-1}\right] \cong E_{i+j} \times E_{i} \cong \mathrm{GL}_{m_{i+j}}(F) \times \mathrm{GL}_{m_{i}}(F)
$$

we have

$$
N_{i, j, 2 k-1} / N_{j, 2 k-1} \cong M_{m_{i+j} m_{i}}(F),
$$

where the action is given by

$$
(X, Y) \cdot A=X A Y^{-1}, \quad X \in G L_{m_{i+j}}(F), A \in M_{m_{i+j} m_{i}}(F), Y \in \mathrm{GL}_{m_{i}}(F) .
$$


Proof. By Theorem 6.19 the only non-trivial factors of the series $\left(N_{j, 2 k-1} / G\left[V_{\infty}\right]\right)_{1 \leq j, 0 \leq k}$ are of the form $\operatorname{Hom}_{F}\left(S_{2 k+1}^{i}, S_{1}^{i+j}\right)$, where $1 \leq j<t-1,0 \leq k \leq k(j), i \in I(j, k)$. From Theorem 5.1 we know that $N$ acts trivially on all this factors, that $E_{l}$ also acts trivially on them if $l \neq i, i+j$, and that $E_{i+j} \times E_{i}$ acts irreducibly as indicated. Since such factor appears as many times as $k$ is between 0 and $s_{i}-s_{i+j}$, the result follows.

\section{The split extension $G\left[V_{\infty}\right]$ of $G\left[V_{\infty}\right] \cap G\left[{ }^{\infty} V / V_{\infty}\right]$}

7.1 Theorem The canonical restriction map $G\left[V_{\infty}\right] \rightarrow G\left({ }^{\infty} V / V_{\infty}\right)$ is a split group epimorphism with kernel $G\left[V_{\infty}\right] \cap G\left[{ }^{\infty} V / V_{\infty}\right]$ and complement $G\left(V_{\text {even }}\right) \times G\left(V_{\text {ndeg }}\right)$. Moreover, $U$ normalizes $E$, so that

$$
\begin{gathered}
G\left\{V_{\text {odd }}^{\dagger}\right\} \cap G\left(V_{\text {odd }}\right)=U \rtimes E, \\
G=G\left[V_{\infty}\right] \rtimes(U \rtimes E),
\end{gathered}
$$

and

$$
G=\left(G\left[V_{\infty}\right] \cap G\left[^{\infty} V / V_{\infty}\right]\right) \rtimes\left(G\left(V_{\text {even }}\right) \times G\left(V_{\text {ndeg }}\right) \times(U \rtimes E)\right)
$$

where the structure of $U$ as a group under the action of $E$ is described in section 6 .

Proof. We know from [3] that $G\left({ }^{\infty} V / V_{\infty}\right)$ preserves the even and non-degenerate parts of ${ }^{\infty} V / V_{\infty}$. It follows that the restriction map

$$
G\left(V_{\text {even }}\right) \times G\left(V_{\text {ndeg }}\right) \hookrightarrow G\left[V_{\infty}\right] \rightarrow G\left({ }^{\infty} V / V_{\infty}\right)
$$

is a group isomorphism. Since

$$
G\left(V_{\text {even }}\right) \times G\left(V_{\text {ndeg }}\right) \cap\left(G\left[V_{\infty}\right] \cap G\left[^{\infty} V / V_{\infty}\right]\right)=1,
$$

we infer

$$
G\left[V_{\infty}\right]=\left(G\left[V_{\infty}\right] \cap G\left[{ }^{\infty} V / V_{\infty}\right]\right) \rtimes\left(G\left(V_{\text {even }}\right) \times G\left(V_{\text {ndeg }}\right)\right) .
$$

The very characterizations of $E$ and $U$ given in Theorems 4.4 and 6.10 show that $E$ normalizes $U$, and both groups are contained in $G\left\{V_{\text {odd }}^{\dagger}\right\} \cap G\left(V_{\text {odd }}\right)$. Moreover, it is obvious 
that $G\left(V_{\text {even }}\right) \times G\left(V_{\text {ndeg }}\right)$ commutes elementwise with $G\left(V_{\text {odd }}\right)$. Furthermore, from Lemma 3.1 we deduce

$$
G\left[V_{\infty}\right] \cap G\left\{V_{\text {odd }}^{\dagger}\right\} \cap G\left(V_{\text {odd }}\right)=1 .
$$

The desired conclusion now follows from Theorems 4.4 and 6.10 .

\section{A criterion applicable to bilinear spaces of types $\mathrm{E}$ and I}

We next derive a criterion that yields the structure of the isometry group of a bilinear space of type E, i.e. the space is equal to its even part, or type I, in C. Riehm's notation.

8.1 Notation If $Y$ is an $F$-vector space and $u \in \operatorname{End}(Y)$ then $C_{\mathrm{GL}(Y)}(u)$ denotes the centralizer of $u$ in $\operatorname{GL}(Y)$.

8.2 Notation If $Y$ and $Z$ are $F$-vector spaces then $\operatorname{Bil}(Y, Z)$ denotes the $F$-vector space of all bilinear forms $Y \times Z \rightarrow F$. We say that $\phi \in \operatorname{Bil}(Y, Z)$ is non-degenerate if its left and right radicals are equal to (0).

8.3 Theorem Let $(W, \phi)$ be a bilinear space. Suppose there exists $G(W, \phi)$-invariant totally isotropic subspaces $Y$ and $Z$ of $W$ such that $W=Y \oplus Z$ and $\left.\phi\right|_{Z \times Y}$ is nondegenerate. Then

(1) There exists a unique $u \in \operatorname{End}_{F}(Y)$ such that

$$
\phi(y, z)=\phi(z, u y), \quad y \in Y, z \in Z \text {. }
$$

(2) If $g \in G(W, \phi)$ then $\left.g\right|_{Y} \in C_{\mathrm{GL}(Y)}(u)$.

(3) The canonical restriction map $\rho: G(W, \phi) \rightarrow C_{\mathrm{GL}(Y)}(u)$, given by $\left.g \mapsto g\right|_{Y}$, is a group isomorphism.

Proof. Consider the linear map $A: \operatorname{End}_{F}(Y) \rightarrow \operatorname{Bil}(Y, Z)$, given by $u \mapsto \phi_{u}$, where

$$
\phi_{u}(y, z)=\phi(z, u y), \quad y \in Y, z \in Z \text {. }
$$

Since the right radical of $\left.\phi\right|_{Z \times Y}$ is (0), it follows that $A$ is a monomorphism. As the left radical of $\left.\phi\right|_{Z \times Y}$ is also (0), we infer $\operatorname{dim} Y=\operatorname{dim} Z$, whence $\operatorname{dim} \operatorname{End}_{F}(Y)=\operatorname{dim} \operatorname{Bil}(Y, Z)$, 
so $A$ is an isomorphism. In particular, there exists a unique $u \in \operatorname{End}_{F}(Y)$ such that $A(u)=\left.\phi\right|_{Y \times Z}$.

Let $g \in G(W, \phi)$. For $y \in Y$ and $z \in Z$, since both $Y$ and $Z$ are $G(W, \phi)$-invariant, (26) gives

$$
\phi(z, g u y)=\phi\left(g^{-1} z, u y\right)=\phi\left(y, g^{-1} z\right)=\phi(g y, z)=\phi(z, u g y) .
$$

As the right radical of $\left.\phi\right|_{Z \times Y}$ is (0), we deduce $\left.g\right|_{Y} \in C_{\mathrm{GL}(Y)}(u)$.

Let $g \in \operatorname{ker} \rho$. For $y \in Y$ and $z \in Z$ we have

$$
\phi(g z, y)=\phi(g z, g y)=\phi(z, y)
$$

As the left radical of $\left.\phi\right|_{Z \times Y}$ is (0), we obtain $\left.g\right|_{Z}=1_{Z}$. But $W=Y \oplus Z$, so $g=1$. This proves that $\rho$ is injective.

Let $b \in C_{\mathrm{GL}(Y)}(u)$. Consider the linear map $\operatorname{End}_{F}(Z) \rightarrow \operatorname{Bil}(Z, Y)$, given by $c \mapsto \phi^{c}$, where

$$
\phi^{c}(z, y)=\phi(c z, y), \quad y \in Y, z \in Z
$$

As above, this is an isomorphism. In particular, there exists a unique $c \in \operatorname{End}_{F}(Z)$ such that

$$
\phi(c z, y)=\phi\left(z, b^{-1} y\right), \quad y \in Y, z \in Z
$$

As $b \in \mathrm{GL}(V)$ and left radical of $\left.\phi\right|_{Z \times Y}$ is (0), we infer that $c \in \mathrm{GL}(Z)$. We may re-write the above equation in the form

$$
\phi(c z, b y)=\phi(z, y), \quad y \in Y, z \in Z
$$

Let $g=b \oplus c \in \mathrm{GL}(W)$. Let $y_{1}, y_{2} \in Y$ and $z_{1}, z_{2} \in Z$. Since $Y$ and $Z$ are totally isotropic, (26) and (27) along with $b \in C_{\mathrm{GL}(Y)}(u)$ give

$$
\begin{aligned}
\phi\left(g\left(y_{1}+z_{1}\right), g\left(y_{2}+z_{2}\right)\right) & =\phi\left(b y_{1}+c z_{1}, b y_{2}+c z_{2}\right)=\phi\left(b y_{1}, c z_{2}\right)+\phi\left(c z_{1}, b y_{2}\right) \\
& =\phi\left(c z_{2}, u b y_{1}\right)+\phi\left(z_{1}, y_{2}\right)=\phi\left(c z_{2}, b u y_{1}\right)+\phi\left(z_{1}, y_{2}\right) \\
& =\phi\left(z_{2}, u y_{1}\right)+\phi\left(z_{1}, y_{2}\right)=\phi\left(y_{1}, z_{2}\right)+\phi\left(z_{1}, y_{2}\right) \\
& =\phi\left(y_{1}+z_{1}, z_{2}\right)+\phi\left(y_{1}+z_{1}, y_{2}\right)=\phi\left(y_{1}+z_{1}, y_{2}+z_{2}\right) .
\end{aligned}
$$

Therefore $g \in G(W, \phi)$. By construction $\rho(g)=b$, so $\rho$ is an epimorphism, thus completing the proof. 
8.4 Note Observe that $Z^{*}$, the dual of $Z$, is an $F G(W, \phi)$-module in the usual way. Moreover, $Y$ and $Z^{*}$ are isomorphic, as $F G(W, \phi)$-modules, via the map $y \mapsto \phi(-, y)$.

\subsection{Structure of $G\left(V_{\text {even }}\right)$}

8.5 Theorem Suppose that $V=V_{\text {even }}$ Then

(1) For uniquely determined positive integers $n_{i}$ and $r_{i}$ we have an equivalence of bilinear spaces

$$
V \cong \underset{1 \leq i \leq d}{\perp} n_{i} N_{2 r_{i}}
$$

(2) There exists a unique $u \in \operatorname{End}_{F}\left(L_{\infty}(V)\right)$ such that

$$
\varphi(l, r)=\varphi(r, u l), \quad l \in L_{\infty}(V), r \in R_{\infty}(V) .
$$

The endomorphism $u$ is nilpotent, with elementary divisors $t^{r_{1}}, \ldots, t^{r_{d}}$ and multiplicities $n_{1}, \ldots, n_{d}$.

(3) The canonical restriction map $\rho: G(V) \rightarrow C_{\mathrm{GL}\left(L_{\infty}(V)\right)}(u)$, given by $\left.g \mapsto g\right|_{L_{\infty}(V)}$, is a group isomorphism.

Proof. The first assertion follows from Theorem 2.1. By means of Lemmas 3.5 and 3.6 we deduce that the subspaces $L_{\infty}(V)$ and $R_{\infty}(V)$ of $V$ satisfy the hypotheses of Theorem 8.3. This theorem yields all remaining assertions, except for the similarity type of $u$. By hypothesis there is a basis of $V$ relative to which the matrix of $\varphi$ is equal to $\underset{1 \leq i \leq d}{\oplus} n_{i} J_{2 r_{i}}(0)$. A suitable rearrangement of this basis which puts first all basis vectors of $R_{\infty}(V)$ and second all basis vectors of $L_{\infty}(V)$ yields a new basis relative to which the matrix of $\varphi$ is equal to

$$
\left(\begin{array}{ll}
0 & 1 \\
J & 0
\end{array}\right),
$$

where $J=\underset{1 \leq i \leq d}{\oplus} n_{i} J_{r_{i}}(0)$. Since $J$ is the matrix of $u$ in the above basis of $L_{\infty}(V)$, the similarity type of $u$ is as given. 


\subsection{Irreducible constituents of the $F G\left(V_{\text {even }}\right)$-module $V_{\text {even }}$}

We know from Note 8.4 that $R_{\infty}(V)^{*} \cong L_{\infty}(V)$, as $F G$-modules. In view this, the decomposition $V=L_{\infty}(V) \oplus R_{\infty}(V)$ and Theorem 8.5, it suffices to restrict ourselves to the classical case of finding the irreducible constituents of $L_{\infty}(V)$ as an $F C_{\mathrm{GL}\left(L_{\infty}(V)\right)}(u)$ module. This is well known and will be omitted.

\section{$9 \quad$ Structure of $G\left(V_{\text {ndeg }}\right)$}

We assume here that $V=V_{\text {ndeg. }}$ Note that $\operatorname{Bil}(V)$ is a natural right $\operatorname{End}(V)$-module via

$$
(\phi \cdot u)(x, y)=\phi(x, u y), \quad \phi \in \operatorname{Bil}(V), u \in \operatorname{End}(V), x, y \in V .
$$

For a fixed $\phi \in \operatorname{Bil}(V)$ the map $\operatorname{End}(V) \rightarrow \operatorname{Bil}(V)$ given by $u \rightarrow \phi \cdot u$ is a linear isomorphism if and only if $\phi$ is non-degenerate, in which case $u$ is invertible if and only if $\phi \cdot u$ is non-degenerate. In this case, given any $\psi \in \operatorname{Bil}(V)$ we shall write $u_{\phi, \psi}$ for the unique $u \in \operatorname{End}(V)$ such that $\psi(x, y)=\phi(x, u y)$.

Since $\varphi$ is non-degenerate, we may use it to represent any bilinear form, in particular $\varphi^{\prime}$. We write $\sigma=u_{\varphi, \varphi^{\prime}}$ for the asymmetry of $\varphi$, i.e. the element of $\operatorname{GL}(V)$ satisfying

$$
\varphi^{\prime}(x, y)=\varphi(x, \sigma(y)), \quad x, y \in V
$$

This linear operator measures how far is $\varphi$ from being symmetric. We have

$$
\varphi(x, y)=\varphi(y, \sigma(x))=\varphi(\sigma(x), \sigma(y)) \quad x, y \in V
$$

so that $\sigma \in G$. In fact, it is easy to see that $\sigma$ belongs to the center $Z(G)$ of $G$.

Let $F[t]$ denote the polynomial algebra in one variable $t$ over $F$. We view $V$ as an $F[t]$-module via $\sigma$. For $0 \neq q \in F[t]$, consider the adjoint polynomial $q^{*} \in F[t]$, defined by

$$
q^{*}(t)=t^{\operatorname{deg} q} q(1 / t)
$$

The minimal polynomial of $\sigma$ will be denoted by $p_{\sigma} \in F[t]$. Let $\mathcal{P}$ stand for the set of all monic irreducible polynomials in $F[t]$ dividing $p_{\sigma}$. For $p \in \mathcal{P}$ let $V_{p}$ denote the primary 
component of $\sigma$ associated to $p$. Since $\sigma \in Z(G)$, each primary component is $G$-invariant. We consider the subsets of $\mathcal{P}$ :

$$
\mathcal{P}_{1}=\left\{p \in \mathcal{P} \mid p^{*} \neq \pm p\right\} \text { and } \mathcal{P}_{2}=\left\{p \in \mathcal{P} \mid p^{*}= \pm p\right\}
$$

We construct a subset $\mathcal{P}_{1}^{\prime}$ of $\mathcal{P}_{1}$ by selecting one element out of each set $\mathcal{P}_{1} \cap\left\{ \pm p, \pm p^{*}\right\}$, as $p$ ranges through $\mathcal{P}_{1}$. It follows at once from [7] that

$$
G(V) \cong\left(\prod_{p \in \mathcal{P}_{1}^{\prime}} G\left(V_{p} \oplus V_{p^{*}}\right)\right) \Pi\left(\prod_{p \in \mathcal{P}_{2}} G\left(V_{p}\right)\right)
$$

Thus the study of $G$ reduces to two cases:

CASE I: $V=V_{p} \oplus V_{p^{*}}, p^{*} \neq \pm p$.

CASE II: $V=V_{p}, p^{*}= \pm p$.

We break II up into two cases:

CASE IIa: $\operatorname{deg} p>1$ or $\operatorname{char} F \neq 2$.

CASE IIb: $\operatorname{deg} p=1$ and $\operatorname{char} F=2$.

\section{$9.1 \quad$ Case I}

We assume here that $p$ is a monic irreducible polynomial in $F[t]$ dividing $p_{\sigma}$ such that $p^{*} \neq \pm p$ and $V=V_{p} \oplus V_{p^{*}}$. In particular, $\left(p, p^{*}\right)=1$. As shown in [7] the $G$-invariant $F[t]$-submodules $V_{p}$ and $V_{p^{*}}$ of $V$ are totally isotropic. In view of Theorem 8.3, we have the following result.

9.1 Theorem The restriction map $\rho: G \rightarrow C_{\mathrm{GL}\left(V_{p}\right)}\left(\left.\sigma\right|_{V_{p}}\right)$ is an isomorphism.

Note that when $F$ is algebraically closed $p=t-\lambda$ for some $\lambda \in F$ different from 1 and -1 . In this case then $G$ becomes isomorphic to the centralizer of a nilpotent element in the general linear group (as adding a scalar operator does not change the centralizer).

\subsection{Case IIa}

We assume here that $p$ is a monic irreducible polynomial in $F[t]$ dividing $p_{\sigma}$ such that $p^{*}= \pm p$ and $V=V_{p}$. We further assume that $\operatorname{deg} p>1$ or char $F \neq 2$. The symmetric 
and alternating parts of $\varphi$ are defined by $\varphi^{ \pm}=\varphi \pm \varphi^{\prime}$. Clearly $G(\varphi) \subseteq G\left(\varphi^{+}\right) \cap G\left(\varphi^{-}\right)$, with equality if $\operatorname{char} F \neq 2$.

9.2 Lemma $\varphi^{ \pm}$is non-degenerate if and only if $p_{\sigma}(\mp 1) \neq 0$.

Proof. This follows from the identity

$$
\varphi^{ \pm}(x, y)=\varphi(x,(1 \pm \sigma) y), \quad x, y \in V
$$

If $\varphi^{+}$is non-degenerate, we write $\sigma^{+-}=u_{\varphi^{+}, \varphi^{-}}$and $\sigma^{+}=u_{\varphi^{+}, \varphi}$; moreover, we denote the isometry group of $\varphi^{+}$by $\mathrm{O}\left(\varphi^{+}\right)$and the associated Lie algebra by $\mathfrak{o}\left(\varphi^{+}\right)$. If $\varphi^{-}$is nondegenerate, we write $\sigma^{-+}=u_{\varphi^{-}, \varphi^{+}}$and $\sigma^{-}=u_{\varphi^{-}, \varphi}$; moreover, we denote the isometry group of $\varphi^{-}$by $\operatorname{Sp}\left(\varphi^{-}\right)$and the associated Lie algebra by $\mathfrak{s p}\left(\varphi^{+}\right)$.

9.3 Lemma If $\varphi^{+}$is non-degenerate then $\sigma^{+-} \in \mathfrak{o}\left(\varphi^{+}\right)$. If $\varphi^{-}$is non-degenerate then $\sigma^{-+} \in \mathfrak{s p}\left(\varphi^{-}\right)$.

Proof. We have

$\varphi^{+}\left(\sigma^{+-} x, y\right)+\varphi^{+}\left(x, \sigma^{+-} y\right)=\varphi^{+}\left(y, \sigma^{+-} x\right)+\varphi^{-}(x, y)=\varphi^{-}(y, x)+\varphi^{-}(x, y)=0, \quad x, y \in V$, thereby proving the first assertion. The second is proved similarly.

9.4 Proposition Suppose $\varphi^{+}$is non-degenerate. Then

$$
G(\varphi)=C_{\mathrm{O}\left(\varphi^{+}\right)}\left(\sigma^{+}\right)
$$

and if $\operatorname{char} F \neq 2$ then

$$
G(\varphi)=C_{\mathrm{O}\left(\varphi^{+}\right)}\left(\sigma^{+-}\right)
$$

Proof. Let $a \in \mathrm{GL}(V)$. We have

$$
a \in G(\varphi)
$$

if and only if

$$
\varphi(a x, a y)=\varphi(x, y), \quad x, y \in V
$$

if and only if

$$
\varphi^{+}\left(a x, \sigma^{+} a y\right)=\varphi^{+}\left(x, \sigma^{+} y\right), \quad x, y \in V
$$


if and only if

$$
\varphi^{+}\left(x, a^{-1} \sigma^{+} a y\right)=\varphi^{+}\left(x, \sigma^{+} y\right), x, y \in V \text { and } a \in \mathrm{O}\left(\varphi^{+}\right)
$$

if and only if

$$
a \in C_{\mathrm{O}\left(\varphi^{+}\right)}\left(\sigma^{+}\right) .
$$

This proves the first assertion. As for the second, if char $F \neq 2$ then

$$
a \in G(\varphi)
$$

if and only if

$$
a \in \mathrm{O}\left(\varphi^{+}\right) \text {and } a \in G\left(\varphi^{-}\right)
$$

if and only if

$$
a \in \mathrm{O}\left(\varphi^{+}\right) \text {and } \varphi^{-}(a x, a y)=\varphi^{-}(x, y), \quad x, y \in V
$$

if and only if

$$
a \in \mathrm{O}\left(\varphi^{+}\right) \text {and } \varphi^{+}\left(a x, \sigma^{+-} a y\right)=\varphi^{+}\left(x, \sigma^{+-} y\right), \quad x, y \in V
$$

if and only if

$$
a \in \mathrm{O}\left(\varphi^{+}\right) \text {and } \varphi^{+}\left(x, a^{-1} \sigma^{+-} a y\right)=\varphi^{+}\left(x, \sigma^{+-} y\right), \quad x, y \in V
$$

if and only if

$$
a \in C_{\mathrm{O}\left(\varphi^{+}\right)}\left(\sigma^{+-}\right) .
$$

9.5 Proposition Suppose $\varphi^{-}$is non-degenerate. Then

$$
G(\varphi)=C_{\operatorname{Sp}\left(\varphi^{-}\right)}\left(\sigma^{-}\right)
$$

and if $\operatorname{char} F \neq 2$ then

$$
G(\varphi)=C_{\operatorname{Sp}\left(\varphi^{-}\right)}\left(\sigma^{-+}\right)
$$

Proof. This is similar to the above proof, mutatis mutandi.

We know from [7] that if $\operatorname{deg} p>1$ then $\operatorname{deg} p$ is even and $p=p^{*}$, while it is obvious that if $\operatorname{deg} p=1$ then $p=t \pm 1$. 
9.6 Theorem (i) If $\operatorname{deg} p>1$ then $\varphi^{ \pm}$is non-degenerate, $\sigma^{+-} \in \mathfrak{o}\left(\varphi^{+}\right), \sigma^{-+} \in \mathfrak{s p}\left(\varphi^{-}\right)$ and

$$
G=C_{\mathrm{O}\left(\varphi^{+}\right)}\left(\sigma^{+}\right)=C_{\mathrm{Sp}\left(\varphi^{-}\right)}\left(\sigma^{-}\right) .
$$

Moreover, if char $F \neq 2$ then

$$
G=C_{\mathrm{O}\left(\varphi^{+}\right)}\left(\sigma^{+-}\right)=C_{\mathrm{Sp}\left(\varphi^{-}\right)}\left(\sigma^{-+}\right)
$$

(ii) If $p=t-1$ and char $F \neq 2$ then $\varphi^{+}$is non-degenerate, $\sigma^{+-} \in \mathfrak{o}\left(\varphi^{+}\right)$and

$$
G=C_{\mathrm{O}\left(\varphi^{+}\right)}\left(\sigma^{+}\right)=C_{\mathrm{O}\left(\varphi^{+}\right)}\left(\sigma^{+-}\right) .
$$

(iii) If $p=t+1$ and char $F \neq 2$ then $\varphi^{-}$is non-degenerate, $\sigma^{-+} \in \mathfrak{s p}\left(\varphi^{-}\right)$and

$$
G=C_{\mathrm{Sp}\left(\varphi^{-}\right)}\left(\sigma^{-}\right)=C_{\mathrm{Sp}\left(\varphi^{-}\right)}\left(\sigma^{-+}\right)
$$

Proof. This follows from Lemmas 9.2 and 9.3, and Propositions 9.4 and 9.5.

For the remainder of this subsection we suppose that $F$ is algebraically closed of characteristic not 2 . Then $p=t \pm 1$.

For convenience, we define $n$-by- $n$ matrices $H_{n}(\lambda)$ and $\Gamma_{n}$ by

$$
H_{n}(\lambda)=\left(\begin{array}{cc}
0 & I_{m} \\
J_{m}(\lambda) & 0
\end{array}\right), \quad n=2 m, \lambda \in F,
$$

and

$$
\Gamma_{n}=\left(\begin{array}{cccccccc}
0 & 0 & 0 & 0 & \cdots & 0 & 0 & (-1)^{n-1} \\
0 & 0 & 0 & 0 & \cdots & 0 & (-1)^{n-2} & (-1)^{n-2} \\
\vdots & & & & & & & \\
0 & -1 & -1 & 0 & \cdots & 0 & 0 & 0 \\
1 & 1 & 0 & 0 & \cdots & 0 & 0 & 0
\end{array}\right)
$$

We refer the reader to [9], and for an older version, [1], for a proof of the Canonical Form Theorem for bilinear forms.

9.7 Theorem (a) Any $\phi \in \operatorname{Bil}(V)$ admits an orthogonal direct decomposition

$$
\phi=\phi_{1} \perp \phi_{2} \perp \cdots \perp \phi_{k},
$$


where the $\phi_{i}$ 's are indecomposable bilinear forms which are unique up to equivalence and permutation.

(b) If $\phi \in \operatorname{Bil}(V)$ is indecomposable then, with respect to a suitable basis of $V$, the matrix of $\phi$ is one of the following:

(i) $H_{n}(\lambda), n=2 m, \lambda \neq(-1)^{m+1}$;

(ii) $\Gamma_{n}, n \geq 1$;

(iii) $J_{n}(0), n=2 m+1$.

(c) The matrices listed in part (b) are pairwise non-congruent except for the fact that $H_{n}(\lambda)$ and $H_{n}\left(\lambda^{-1}\right)$ are congruent when $\lambda \neq 0, \pm 1$.

We mention that, when $n=2 m$ is even, $H_{n}(0)$ is congruent to $J_{n}(0)$.

9.8 Theorem If $p=t-1$ then $\varphi^{+}$is non-degenerate, $\sigma^{+-}$belongs to $\mathfrak{s o}\left(\varphi^{+}\right), G=$ $C_{\mathrm{O}\left(\varphi^{+}\right)}\left(\sigma^{+-}\right)$, and the linear operators $\sigma-1$ and $\sigma^{+-}$are nilpotent and similar to each other (i.e., they have the same elementary divisors).

Proof. In view of Theorem 9.6 we are reduced to show the last assertion. It suffices to verify this assertion for indecomposable $\varphi$. There are two cases to consider. The matrix of $\varphi$ will be denoted by $A_{\varphi}$.

First, the matrix of $\varphi$ is $H_{n}(1)$ where $n=2 m$ and $m$ is even. Then the matrix of $u$ is $-A_{\varphi^{+}}^{-1} A_{\varphi^{-}}$. An easy computation shows that both $\sigma-1$ and $\sigma^{+-}$have elementary divisors $t^{m}$ and $t^{m}$.

Second, the matrix of $\varphi$ is $\Gamma_{n}$ and $n$ is odd. In that case the matrix $A_{\varphi^{+}}$is involutory and a simple computation shows that the matrix of $\sigma^{+-}$is equal to $-J_{n}(0)^{\prime}$. Hence both $\sigma-1$ and $\sigma^{+-}$have only one elementary divisor, namely $t^{n}$.

9.9 Theorem If $p=t+1$ then $\varphi^{-}$is non-degenerate, $\sigma^{-+}$belongs to $\mathfrak{s p}\left(\varphi^{-}\right), G=$ $C_{\mathrm{Sp}\left(\varphi^{-}\right)}\left(\sigma^{-+}\right)$, and the linear operators $\sigma+1$ and $\sigma^{-+}$are nilpotent and similar to each other (i.e., they have the same elementary divisors).

Proof. This proof is similar to the one above. 


\subsection{Case IIb}

We have not been able to make progress on this case.

\section{The 2-step nilpotent group $G\left[V_{\infty}\right] \cap G\left[{ }^{\infty} V / V_{\infty}\right]$}

The divide the study of $G\left[V_{\infty}\right] \cap G\left[{ }^{\infty} V / V_{\infty}\right]$ into two cases, namely that of $G\left[{ }^{\infty} V\right]$ and $G\left[V_{\infty}\right] \cap G\left[{ }^{\infty} V / V_{\infty}\right] / G\left[{ }^{\infty} V\right]$.

\subsection{Basic facts about $G\left[V_{\infty}\right] \cap G\left[{ }^{\infty} V / V_{\infty}\right]$}

10.1 Lemma $G\left[{ }^{\infty} V\right]$ is contained in the center of $G\left[V_{\infty}\right] \cap G\left[{ }^{\infty} V / V_{\infty}\right]$.

Proof. From Lemma 3.2 we know that $G\left[{ }^{\infty} V\right]$ is contained in $G\left[V_{\infty}\right] \cap G\left[V / V_{\infty}\right]$, which is in turn contained in $G\left[V_{\infty}\right] \cap G\left[{ }^{\infty} V / V_{\infty}\right]$. Let $g \in G\left[{ }^{\infty} V\right]$ and $h \in G\left[V_{\infty}\right] \cap G\left[{ }^{\infty} V / V_{\infty}\right]$. By Lemma 3.2 we have $(g-1) V \subseteq V_{\infty}$. Since $G\left[V_{\infty}\right] \cap G\left[{ }^{\infty} V / V_{\infty}\right]$ is the identity on $V_{\infty}$, we infer $(h-1)(g-1) V=(0)$. Moreover, $(h-1) V \subseteq{ }^{\infty} V$ by Lemma 3.1, whence $(g-1)(h-1) V=0$ by the very definition of $G\left[{ }^{\infty} V\right]$. It follows that

$$
h g=h+g-1=g h,
$$

as required.

10.2 Lemma $G\left[V_{\infty}\right] \cap G\left[{ }^{\infty} V / V_{\infty}\right]$ is nilpotent of class $\leq 2$.

Proof. By Lemma 10.1 it suffices to show that $G\left[V_{\infty}\right] \cap G\left[{ }^{\infty} V / V_{\infty}\right] / G\left[{ }^{\infty} V\right]$ is abelian. Let $g, h \in G\left[V_{\infty}\right] \cap G\left[{ }^{\infty} V / V_{\infty}\right]$ and let $v \in{ }^{\infty} V$. Since $g v-v \in V_{\infty}$, by the definition of $G\left[V_{\infty}\right] \cap G\left[{ }^{\infty} V / V_{\infty}\right]$, and $h$ is the identity on $V_{\infty}$, we have

$$
h(g(v))=h(g(v)-v+v)=g v-v+h v .
$$

For the same reasons as above

$$
\left(h^{-1} g^{-1} h g\right)(v)=h^{-1} g^{-1}(g v+h v-v)=h^{-1}(v+h v-v)=h^{-1} h v=v .
$$

Thus $[h, g] \in G\left[{ }^{\infty} V\right]$, as required. 
10.3 Lemma $G\left[V_{\infty}\right] \cap G\left[V / V_{\infty}\right]$ is an abelian unipotent normal subgroup of $G$. In fact, if $g, h \in G\left[V_{\infty}\right] \cap G\left[V / V_{\infty}\right]$ then

$$
(h-1)(g-1)=0 .
$$

Proof. Since $V_{\infty}$ is $G$-invariant, it follows that $G\left[V_{\infty}\right] \cap G\left[V / V_{\infty}\right]$ is a normal subgroup of $G$. If if $g, h \in G\left[V_{\infty}\right] \cap G\left[V / V_{\infty}\right]$ then $(g-1) V \subseteq V_{\infty}$, so $(h-1)(g-1) V=0$. This completes the proof.

10.4 Lemma $G\left[V_{\infty}\right] \cap G\left[{ }^{\infty} V / V_{\infty}\right]$ is a unipotent normal subgroup of $G$. In fact, if $g, h, k \in G\left[V_{\infty}\right] \cap G\left[{ }^{\infty} V / V_{\infty}\right]$ then $(k-1)(h-1)(g-1)=(0)$.

Proof. Since ${ }^{\infty} V$ and $V_{\infty}$ are $G$-invariant, it follows that $G\left[V_{\infty}\right] \cap G\left[{ }^{\infty} V / V_{\infty}\right]$ is a normal subgroup of $G$. Let $g, h, k \in G\left[V_{\infty}\right] \cap G\left[{ }^{\infty} V / V_{\infty}\right]$. By Lemma 3.1 we have $(g-1) V \subseteq{ }^{\infty} V$. From the very definition of $G\left[V_{\infty}\right] \cap G\left[{ }^{\infty} V / V_{\infty}\right]$ we obtain $(h-1)(g-1) V \subseteq V_{\infty}$ and a fortiori $(k-1)(h-1)(g-1) V=(0)$. This completes the proof.

\subsection{Structure of the $F G$-module $G\left[{ }^{\infty} V\right] \cap G[V / \operatorname{Rad}(V)]$}

The divide the study of $G\left[^{\infty} V\right]$ into two cases, namely that of $G\left[{ }^{\infty} V\right] \cap G[V / \operatorname{Rad}(V)]$ and $G\left[{ }^{\infty} V\right] / G\left[{ }^{\infty} V\right] \cap G[V / \operatorname{Rad}(V)]$.

10.5 Theorem The map $G\left[{ }^{\infty} V\right] \rightarrow \operatorname{End}_{F}(V)$ given by

$$
g \mapsto g-1
$$

is a group monomorphism whose image is an $F$-vector subspace of $\operatorname{End}_{F}(V)$. By transferring this $F$-vector space structure to $G\left[V_{\infty}\right] \cap G\left[V / V_{\infty}\right]$ the map (31) becomes an $F G$ module monomorphism. The map (31) induces a monomorphism of FG-modules

$$
G\left[^{\infty} V\right] \rightarrow \operatorname{Hom}_{F}\left(V /^{\infty} V, V_{\infty}\right)
$$

and hence a monomorphism of $F$-vector spaces

$$
\left.G{ }^{\infty} V\right] \rightarrow \operatorname{Hom}_{F}\left(V_{\text {odd }}^{\dagger}, V_{\infty}\right)
$$

namely by means of $\left.g \mapsto(g-1)\right|_{V_{\text {odd }}^{\dagger}}$. 
Proof. The identity of (30) shows that (31) is a group homomorphism, which is clearly injective and preserves the action of $G$. Suppose $g \in G\left[^{\infty} V\right]$ and $k \in F$. Then $k(g-1)+1$ is a linear automorphism of $V$ which fixes ${ }^{\infty} V$ pointwise, acts trivially on $V / V_{\infty}$ and preserves the orthogonality of the generators $e_{2 k}^{i, p}$ of $V_{\text {odd }}^{\dagger}$. It follows that $k(g-1)+1 \in G\left[{ }^{\infty} V\right]$, so the image of (31) is a subspace of $\operatorname{End}_{F}(V)$. By Lemma 3.2 we know that (31) maps $G\left[{ }^{\infty} V\right]$ into $\operatorname{Hom}_{F}\left(V, V_{\infty}\right)$, and the very definition of $G\left[{ }^{\infty} V\right]$ yields an induced $F G$-monomorphism $G\left[^{\infty} V\right] \rightarrow \operatorname{Hom}_{F}\left(V /{ }^{\infty} V, V_{\infty}\right)$. Since $V_{\text {odd }}^{\dagger}$ complements ${ }^{\infty} V$ in $V$, the last assertion follows.

10.6 Lemma $\quad G\left[{ }^{\infty} V\right] \cap G[V / L(V)]=G\left[^{\infty} V\right] \cap G[V / \operatorname{Rad}(V)]$.

Proof. By definition the right hand side is contained in the left hand side. Let $g \in$ $G\left[^{\infty} V\right] \cap G[V / L(V)]$. We wish to show that $(g-1) V \subseteq \operatorname{Rad}(V)$. Since $g$ is the identity on ${ }^{\infty} V$, it suffices to prove $(g-1) V_{\text {odd }}^{\dagger} \subseteq \operatorname{Rad}(V)$. By assumption $(g-1) V_{\text {odd }}^{\dagger} \subseteq L(V)$, so are reduced to demonstrate $(g-1) V_{\text {odd }}^{\dagger} \subseteq R(V)$. By Lemma 3.2 we have $(g-1) V_{\text {odd }}^{\dagger} \subseteq V_{\infty}$, which leave only the identity $\left\langle V_{\text {odd }}^{\dagger},(g-1) V_{\text {odd }}^{\dagger}\right\rangle=0$ to be shown. Well, if $v, w \in V_{\text {odd }}^{\dagger}$ then $g w-w \in L(V)$, so

$$
0=\langle w, v\rangle=\langle g w, g v\rangle=\langle g w, g v\rangle=\langle(g w-w)+w, g v\rangle=\langle w, g v\rangle=\langle w, g v-v\rangle
$$

as required.

10.7 Definition Let $\operatorname{Bil}\left(V,^{\infty} V\right)$ be the $F G$-submodule of $\operatorname{Bil}(V)$ consisting of all bilinear forms whose radical contains ${ }^{\infty} V$. Thus $\operatorname{Bil}\left(V,{ }^{\infty} V\right)$ and $\operatorname{Bil}\left(V /{ }^{\infty} V\right)$ are isomorphic as $F G$-modules.

10.8 Theorem The map $G\left[^{\infty} V\right] \rightarrow \operatorname{Bil}\left(V,{ }^{\infty} V\right)$ given by $g \mapsto \varphi_{g}$, where

$$
\varphi_{g}(v, w)=\varphi((g-1) v, w)=\langle(g-1) v, w\rangle, \quad v, w \in V
$$

is an $F G$-module homomorphism, inducing an $F G$-module homomorphism $g \mapsto \widehat{\varphi_{g}}$ from $G\left[^{\infty} V\right]$ to $\operatorname{Bil}\left(V /{ }^{\infty} V\right)$. The kernel of both maps is equal to $G\left[^{\infty} V\right] \cap G[V / \operatorname{Rad}(V)]$.

Proof. The fact that $g \mapsto \varphi_{g}$ is a homomorphism of $F G$-modules is easily verified. By the very definition of this map its kernel is equal to $G\left[^{\infty} V\right] \cap G[V / L(V)]$, which equals $G\left[{ }^{\infty} V\right] \cap G[V / \operatorname{Rad}(V)]$ by Lemma 10.6 . 
10.9 Notation The image of $G\left[{ }^{\infty} V\right]$ under the above $F G$-homomorphism $G\left[{ }^{\infty} V\right] \rightarrow$ $\operatorname{Bil}(V / \infty V)$ will be denoted by $S$.

10.10 Lemma The restriction of (31) to $G\left[{ }^{\infty} V\right] \cap G[V / \operatorname{Rad}(V)]$ yields an isomorphism of $F G$-modules

$$
G\left[^{\infty} V\right] \cap G[V / \operatorname{Rad}(V)] \rightarrow \operatorname{Hom}_{F}\left(V /{ }^{\infty} V, \operatorname{Rad}(V)\right)
$$

Proof. All maps of the form $1_{\infty} \oplus\left(1_{V_{\text {odd }}^{\dagger}}+f\right)$, where $f \in \operatorname{Hom}_{F}\left(V_{\text {odd }}^{\dagger}, \operatorname{Rad}(V)\right)$, belong to $G\left[{ }^{\infty} V\right] \cap G[V / \operatorname{Rad}(V)]$, thereby proving that (33) is an epimorphism. The rest follows from Theorem 10.5.

10.11 Theorem $\quad G\left[^{\infty} V\right] \cap G[V / \operatorname{Rad}(V)]$ is an $F G$-module of dimension $\left(\operatorname{dim} V /{ }^{\infty} V\right) \times$ $(\operatorname{dim} \operatorname{Rad}(V))$. If $\operatorname{Rad}(V) \neq 0$ and $V /{ }^{\infty} V \neq 0$ its irreducible constituents are of the form $\operatorname{Hom}_{F}\left(Q_{2 k}^{i}, \operatorname{Rad}(V)\right)$, where the $Q_{2 k}^{i}$ are the irreducible constituents of the $F G$-module $V / \infty V$ described in Theorem 6.15. Each has dimension $m_{i} m_{t}, 1 \leq i<t$, and multiplicity $s_{i}$ with stabilizer $S_{i}=N \rtimes \prod_{l \neq i, t} E_{l}$. As a module for $G / S_{i} \cong E_{i} \times E_{t} \cong \mathrm{GL}_{m_{i}}(F) \times \mathrm{GL}_{m_{t}}(F)$, $\operatorname{Hom}_{F}\left(Q_{2 k}^{i}, \operatorname{Rad}(V)\right)$ is isomorphic to $M_{m_{t} m_{i}}(F)$, where $(X, Y) \in \mathrm{GL}_{m_{i}}(F) \times \mathrm{GL}_{m_{t}}(F)$ acts on $A \in M_{m_{t} m_{i}}(F)$ by $(X, Y) \cdot A=Y A X^{-1}$.

Proof. This follows easily from Lemma 10.10 and Theorem 6.15.

We next wish to determine the structure of the the remaining part of $G\left[{ }^{\infty} V\right]$, namely

$$
G\left[^{\infty} V\right] / G\left[{ }^{\infty} V\right] \cap G[V / \operatorname{Rad}(V)] \cong_{F G} S
$$

We digress to record some basic facts from Linear Algebra which will be required for a complete understanding of the structure of $S$.

\section{3 $\mathrm{GL}_{m}(F)$ acting by congruence on $M_{m}(F)$}

10.12 Definition Let $m \geq 1$. Denote by $S_{m}(F)$ and $A_{m}(F)$ the set of all $m \times m$ symmetric and alternating matrices over $F$, respectively.

10.13 Theorem Let $m \geq 1$. The irreducible constituents of $M_{m}(F)$ as a module for $\mathrm{GL}_{m}(F)$ over $F$, acting by congruence are as follows. 
(1) If char $F \neq 2$ then

$$
M_{m}(F)=S_{m}(F) \oplus A_{m}(F)
$$

where both summands are irreducible if $m>1$, while $M_{1}(F)=S_{1}(F)$ is irreducible.

(2) If char $F=2$ each factor of the $\mathrm{GL}_{m}(F)$-invariant series

$$
0 \subseteq A_{m}(F) \subseteq S_{m}(F) \subseteq M_{m}(F)
$$

is irreducible, except when $m=1$ in which case $M_{1}(F)=S_{1}(F)$ is irreducible.

(3) $M_{m}(F) / S_{m}(F)$ is isomorphic to $A_{m}(F)$.

Proof. Consider the homomorphism of $\mathrm{GL}_{m}(F)$-modules $M_{m}(F) \rightarrow A_{m}(F)$, given by $A \mapsto A-A^{\prime}$. Since its kernel is $S_{m}(F)$, a dimension comparison shows that its image is $A_{m}(F)$. Therefore $M_{m}(F) / S_{m}(F) \cong A_{m}(F)$. In view of this isomorphism we may assume throughout that $m>1$, and we are reduced to show that $A_{m}(F)$ and $S_{m}(F) / A_{m}(F)$ are irreducible, in the later case when char $F=2$.

SteP I: $A_{m}(F)$ is irreducible. If $m=2,3$ there is a single non-zero $\mathrm{GL}_{m}(F)$-orbit in $A_{m}(F)$, which is then irreducible. Suppose $m>3$. Let $0 \neq M$ be any $F \mathrm{GL}_{m}(F)$ submodule of $A_{m}(F)$. To see that $M=A_{m}(F)$ it suffices to show that $M$ contains a matrix of rank 2. It is well-known that $M$ contains a matrix, say $A$, which is the direct sum of at least one block of the form $\left(\begin{array}{cc}0 & 1 \\ -1 & 0\end{array}\right)$ plus zero blocks. We may assume that $A$ has at least two non-zero blocks. Choose $B$ in $A_{m}(F)$ whose only nonzero entries are in positions $(2,3)$ and $(3,2)$. Then $A+B$ has the same rank as $A$ and so $A+B$ is in $M$. Hence $B$ is in $M$, as required.

SteP II: $S_{m}(F) / A_{m}(F)$ is irreducible if char $F=2$. Let $A \in S_{m}(F)$ be a nonalternating matrix. It is well-known $A$ is congruent to a non-zero diagonal matrix, say $D$. Thus, in order to show that that the $\mathrm{GL}_{m}(F)$-submodule of $S_{m}(F)$, say $M$, generated by $A$ and $A_{m}(F)$ is equal to $S_{m}(F)$, it suffices to show that $M$ contains a matrix of rank 1 . Suppose $D$ has rank $>1$; by scaling $D$ we may assume that its first two diagonal entries are equal to 1 . Let $D_{1}$ be the matrix obtained from $D$ by replacing its top left $2 \times 2$ corner by $\left(\begin{array}{ll}0 & 1 \\ 1 & 1\end{array}\right)$ and let $D_{2} \in A_{m}(F)$ be the direct sum of $\left(\begin{array}{ll}0 & 1 \\ 1 & 0\end{array}\right)$ and the zero block. Then 
$D_{1}$ and $D_{2}$ belong to $M$ and $D_{1}-D_{2}$ is a non-zero diagonal matrix whose first entry is 0 . It follows by induction that $M$ contains a matrix of rank 1 , as required.

10.14 Note It might seem that all $\mathrm{GL}_{m}(F)$-submodules of $M_{m}(F)$ can be obtained from above, but there is at least one exception. If $F=F_{2}$ then $S_{2}(F)$ is the direct sum of the 1-dimensional submodule $A_{m}(F)$ with the 2-dimensional submodule generated by the identity matrix.

\subsection{The structure of the $F G$-module $G\left[{ }^{\infty} V\right] /\left(G\left[{ }^{\infty} V\right] \cap G[V / \operatorname{Rad}(V)]\right)$}

We refer the reader to the definition of the $F G$-submodules $(i) V$ of $V$, and the irreducible $F G$-modules $Q_{2 k}^{i}$ built upon them, both of which are defined prior to Theorem 6.15.

10.15 Notation If $U$ and $W$ are $F G$-modules, let $\operatorname{Bil}(U, W)$ denote the $F G$-module of all bilinear forms $U \times W \rightarrow F$.

10.16 Definition For $1 \leq i \leq \mathbf{t}+1$ let

$$
\mathcal{M}_{i}=\left\{\phi \in \operatorname{Bil}\left(V /{ }^{\infty} V\right) \mid(i-1) V /(0) V \subseteq \operatorname{Rad}(\phi)\right\} .
$$

We have a series of $F G$-modules

$$
\operatorname{Bil}\left(V /^{\infty} V\right)=\mathcal{M}_{1} \supset \mathcal{M}_{2} \supset \cdots \supset \mathcal{M}_{\mathbf{t}+1}=(0) .
$$

We further refine each link $\mathcal{M}_{i} \supset \mathcal{M}_{i+1}, 1 \leq i \leq \mathbf{t}$ of this chain as follows.

10.17 Definition For $1 \leq i \leq j \leq \mathbf{t}+1$ let

$\mathcal{L}_{i, j}=\left\{\phi \in \mathcal{M}_{i} \mid \phi((i) V /(0) V,(j-1) V /(0) V)=0\right.$ and $\left.\phi((j-1) V /(0) V,(i) V /(0) V)=0\right\}$.

We have a series of $F G$-modules

$$
\mathcal{M}_{i}=\mathcal{L}_{i, i} \supset \mathcal{L}_{i, i+1} \supset \cdots \supset \mathcal{L}_{i, \mathbf{t}+1}=\mathcal{M}_{i+1}
$$

This yields a refined series of $F G$-modules for $\operatorname{Bil}\left(V /{ }^{\infty} V\right)$, and intersecting each term with $S$ we get a series of $F G$-modules for $S$. Our goal is to further refine this series into a composition series for $S$, with known factors, as described below. 
Let $1 \leq i \leq \mathbf{t}$. The very definition of $\mathcal{M}_{i}$ yields an isomorphism of $F G$-modules

$$
\mathcal{M}_{i} \rightarrow \operatorname{Bil}(V /(i-1) V)
$$

Post-composing (34) with restriction to $(i) V /(i-1) V \times(i) V /(i-1) V$ yields a homomorphism of $F G$-modules

$$
\mathcal{M}_{i} \rightarrow \operatorname{Bil}((i) V /(i-1) V)
$$

whose kernel is precisely $\mathcal{L}_{i, i+1}$. This yields a monomorphism of $F G$-modules

$$
\mathcal{M}_{i} \cap S /\left(\mathcal{L}_{i, i+1} \cap S\right) \rightarrow \operatorname{Bil}((i) V /(i-1) V) .
$$

By restricting to

$$
Q_{2}^{i} \times Q_{2}^{i}, Q_{2}^{i} \times Q_{4}^{i}, \ldots, Q_{2}^{i} \times Q_{2 s_{i}}^{i}
$$

we get a homomorphism of $F G$-modules

$$
\mathcal{M}_{i} \cap S /\left(\mathcal{L}_{i, i+1} \cap S\right) \rightarrow \underset{1 \leq k \leq s_{i}}{\oplus} \operatorname{Bil}\left(Q_{2}^{i}, Q_{2 k}^{i}\right) .
$$

We shall show below that (37) is in fact an isomorphism.

Let $1 \leq i<j \leq \mathbf{t}$. Post-composing (34) with restriction to $(i) V /(i-1) V \times(j) V /(i-1) V$ and $(j) V /(i-1) V \times(i) V /(i-1) V$ yields a homomorphism of $F G$-modules

$$
\mathcal{L}_{i, j} \rightarrow \operatorname{Bil}((i) V /(i-1) V,(j) V /(i-1) V) \oplus \operatorname{Bil}((j) V /(i-1) V,(i) V /(i-1) V) .
$$

By the very nature of $\mathcal{L}_{i, j}$ this yields a homomorphism of $F G$-modules

$$
\mathcal{L}_{i, j} \rightarrow \operatorname{Bil}((i) V /(i-1) V,(j) V /(j-1) V) \oplus \operatorname{Bil}((j) V /(j-1) V,(i) V /(i-1) V),
$$

whose kernel is precisely $\mathcal{L}_{i, j+1}$. This yields a monomorphism of $F G$-modules $\mathcal{L}_{i, j} \cap S /\left(\mathcal{L}_{i, j+1} \cap S\right) \rightarrow \operatorname{Bil}((i) V /(i-1) V,(j) V /(j-1) V) \oplus \operatorname{Bil}((j) V /(j-1) V,(i) V /(i-1) V)$.

By restricting to

$$
Q_{2}^{i} \times Q_{2}^{j}, Q_{2}^{i} \times Q_{4}^{j}, \ldots, Q_{2}^{i} \times Q_{2 s_{j}}^{j}
$$

and

$$
Q_{2}^{j} \times Q_{2}^{i}, Q_{2}^{j} \times Q_{4}^{i}, \ldots, Q_{2}^{j} \times Q_{2 s_{i}}^{i}
$$


we get a homomorphism of $F G$-modules

$$
\mathcal{L}_{i, j} \cap S /\left(\mathcal{L}_{i, j+1} \cap S\right) \rightarrow \underset{1 \leq l \leq s_{j}}{\oplus} \operatorname{Bil}\left(Q_{2}^{i}, Q_{2 l}^{j}\right) \bigoplus_{1 \leq k \leq s_{i}}^{\oplus} \operatorname{Bil}\left(Q_{2}^{j}, Q_{2 k}^{i}\right) .
$$

We shall show below that (41) is in fact an isomorphism.

10.18 Theorem $\quad G\left[^{\infty} V\right] /\left(G\left[^{\infty} V\right] \cap G[V / \operatorname{Rad}(V)]\right)$ is an $F G$-module of dimension

$$
\operatorname{dim} G\left[{ }^{\infty} V\right] /\left(G\left[^{\infty} V\right] \cap G[V / \operatorname{Rad}(V)]\right)=\operatorname{dim}\left(V /{ }^{\infty} V\right)\left(m_{1}+\cdots+m_{\mathbf{t}}\right),
$$

so the $F G$-module $G\left[^{\infty} V\right]$ has dimension $\operatorname{dim}\left(V /{ }^{\infty} V\right)\left(m_{1}+\cdots+m_{t}\right)$.

The irreducible constituents of $G\left[{ }^{\infty} V\right] /\left(G\left[^{\infty} V\right] \cap G[V / \operatorname{Rad}(V)]\right)$ as an $F G$-module are obtained as follows. We start with the series for $S \cong_{F G} G\left[{ }^{\infty} V\right] /\left(G\left[{ }^{\infty} V\right] \cap G[V / \operatorname{Rad}(V)]\right)$ produced after Definition 10.17 and then decompose each factor by means of the maps (37) and (41), both of which are isomorphisms.

Each summand in (41) is an irreducible FG-module, while the summands in (37) has the constituents indicated in Theorem 10.13. More precisely, we have the following situation.

(1) If $1 \leq i \neq j \leq \mathbf{t}$ and $1 \leq l \leq s_{j}$ then the composition factor $\operatorname{Bil}\left(Q_{2}^{i}, Q_{2 l}^{j}\right)$ of $G\left[^{\infty} V\right] / G\left[{ }^{\infty} V\right] \cap G[V / \operatorname{Rad}(V)]$ is $F G$-irreducible,

$$
G\left[\operatorname{Bil}\left(Q_{2}^{i}, Q_{2 l}^{j}\right)\right] \supseteq N \rtimes \prod_{k \neq i, j} E_{k}
$$

where

$$
G /\left(N \rtimes \prod_{k \neq i, j} E_{k}\right) \cong E_{i} \times E_{j} \cong \mathrm{GL}_{m_{i}}(F) \times \mathrm{GL}_{m_{j}}(F)
$$

acts on

$$
\operatorname{Bil}\left(Q_{2}^{i}, Q_{2 l}^{j}\right) \cong M_{m_{i}, m_{j}}(F)
$$

by congruence

$$
(X, Y) \cdot A=X A Y^{\prime}, \quad X \in \mathrm{GL}_{m_{i}}(F), A \in M_{m_{i}, m_{j}}(F), Y \in \mathrm{GL}_{m_{j}}(F)
$$

(2) If $1 \leq i \leq \mathbf{t}$ and $1 \leq k \leq s_{i}$ then the factor $\operatorname{Bil}\left(Q_{2}^{i}, Q_{2 k}^{i}\right)$ of the aforementioned series $G\left[^{\infty} V\right] /\left(G\left[^{\infty} V\right] \cap G[V / \operatorname{Rad}(V)]\right)$ possesses the following properties.

$$
G\left[\operatorname{Bil}\left(Q_{2}^{i}, Q_{2 k}^{i}\right)\right] \supseteq N \rtimes \prod_{l \neq i} E_{l},
$$


where

$$
G /\left(N \rtimes \prod_{l \neq i} E_{l}\right) \cong E_{i} \cong \mathrm{GL}_{m_{i}}(F)
$$

acts on

$$
\operatorname{Bil}\left(Q_{2}^{i}, Q_{2 k}^{i}\right) \cong M_{m_{i}}(F)
$$

by congruence

$$
X \cdot A=X A X^{\prime}, \quad X \in \mathrm{GL}_{m_{i}}(F), A \in M_{m_{i}}(F) .
$$

The irreducible constituents of $\operatorname{Bil}\left(Q_{2}^{i}, Q_{2 k}^{i}\right)$ are therefore as indicated in Theorem 10.13.

Proof. We first establish the inequality

$$
\operatorname{dim} G\left[{ }^{\infty} V\right] /\left(G\left[^{\infty} V\right] \cap G[V / \operatorname{Rad}(V)]\right) \geq\left(\operatorname{dim} V /{ }^{\infty} V\right)\left(m_{1}+\cdots+m_{\mathbf{t}}\right) .
$$

Recall the $F$-linear monomorphism (32). We easily see that a necessary and sufficient condition for $f \in \operatorname{Hom}_{F}\left(V_{\text {odd }}^{\dagger}, V_{\infty}\right)$ to be in its image is that the vectors $e_{2 k}^{i, p}, 1 \leq i \leq \mathbf{t}$, $1 \leq p \leq m_{i}, 1 \leq k \leq s_{i}$ remain $\varphi$-orthogonal under $f+1$. This yields a linear system of

$$
\left(s_{1} m_{1}+\cdots+s_{\mathbf{t}} m_{\mathbf{t}}\right)^{2}
$$

equations in

$$
\left(s_{1} m_{1}+\cdots+s_{\mathbf{t}} m_{\mathbf{t}}\right)\left(\left(s_{1}+1\right) m_{1}+\cdots+\left(s_{t}+1\right) m_{t}\right)
$$

variables. Thus

$$
\operatorname{dim} G\left[^{\infty} V\right] \geq\left(s_{1} m_{1}+\cdots+s_{\mathbf{t}} m_{\mathbf{t}}\right)\left(m_{1}+\cdots+m_{t}\right)=\left(\operatorname{dim} V /{ }^{\infty} V\right)\left(m_{1}+\cdots+m_{t}\right) .
$$

But from Lemma 10.10 we know that

$$
\operatorname{dim} G\left[{ }^{\infty} V\right] \cap G[V / \operatorname{Rad}(V)]=\operatorname{dim} \operatorname{Hom}_{F}\left(V /{ }^{\infty} V, \operatorname{Rad}(V)\right)
$$

By combining (44) and (45) we obtain (43).

We next explicitly describe the linear system governing the image of (32). Let $f \in$ $\operatorname{Hom}_{F}\left(V_{\text {odd }}^{\dagger}, V_{\infty}\right)$ and write

$$
f\left(e_{2 k}^{i, p}\right)=\sum_{1 \leq j \leq t} \sum_{1 \leq q \leq m_{i}} \sum_{0 \leq l \leq s_{i}} i_{j} X_{2 k, 2 l+1}^{p, q} e_{2 l+1}^{j, q},
$$


where ${ }_{i, j} X_{2 k, 2 l+1}^{p, q} \in F$. Then $f=\left.(g-1)\right|_{V_{\text {odd }}^{\dagger}}$ for some $g \in G\left[{ }^{\infty} V\right]$ if and only if for $1 \leq i, j \leq \mathbf{t}, 1 \leq k \leq s_{i}, 1 \leq l \leq s_{j}, 1 \leq p \leq m_{i}$ and $1 \leq q \leq m_{j}$ we have

$$
0=\left\langle(f+1)\left(e_{2 k}^{i, p}\right),(f+1)\left(e_{2 l}^{j, q}\right)\right\rangle={ }_{i, j} X_{2 k, 2 l+1}^{p, q}+{ }_{j, i} X_{2 l, 2 k-1}^{q, p}
$$

We next utilize (46) and (47) to show that equality prevails in (43), and to infer from it that (37) and (41) are isomorphisms.

Suppose first that $1 \leq i \leq \mathbf{t}$ and $\phi \in \operatorname{Bil}((i) V /(i-1) V)$ belongs to the image of (36). From the very definition of $S$ we see that $\phi$ is the image under (35) of $\widehat{\varphi_{g}} \in \mathcal{M}_{i}$ for some $g \in G\left[{ }^{\infty} V\right]$. For $1 \leq k, l \leq s_{i}$ let ${ }_{i} A_{2 k, 2 l} \in M_{m_{i}}(F)$ denote the Gram matrix of $\left.\phi\right|_{Q_{2 k}^{i} \times Q_{2 l}^{i}}$ relative to the bases of $Q_{2 k}^{i}$ and $Q_{2 l}^{i}$ described in Theorem 6.15. For $1 \leq p, q \leq m_{i}$ let ${ }_{i} A_{2 k, 2 l}^{p, q}$ denote the $(p, q)$-entry of ${ }_{i} A_{2 k, 2 l}$. Then

$$
\begin{aligned}
{ }_{i} A_{2 k, 2 l}^{p, q} & \left.=\phi\left(e_{2 k}^{i, p}+(i-1) V, e_{2 l}^{i, q}+(i-1) V\right)\right) \\
& =\widehat{\varphi_{g}}\left(e_{2 k}^{i, p}+{ }^{\infty} V, e_{2 l}^{i, q}+{ }^{\infty} V\right) \\
& =\varphi_{g}\left(e_{2 k}^{i, p}, e_{2 l}^{i, q}\right) \\
& =\varphi\left((g-1) e_{2 k}^{i, p}, e_{2 l}^{i, q}\right)=\left\langle(g-1) e_{2 k}^{i, p}, e_{2 l}^{i, q}\right\rangle .
\end{aligned}
$$

Let $f=\left.(g-1)\right|_{V_{\text {odd }}^{\dagger}}$ and let $(46)$ be the representation of $f$ relative to our chosen basis of $V_{\text {odd }}^{\dagger}$. Then (46) and (48) yield

$$
{ }_{i} A_{2 k, 2 l}^{p, q}=\left\langle(g-1) e_{2 k}^{i, p}, e_{2 l}^{j, q}\right\rangle=\left\langle f\left(e_{2 k}^{i, p}\right), e_{2 l}^{j, q}\right\rangle={ }_{i, i} X_{2 k, 2 l+1}^{p, q}
$$

Applying (49) and (47), we see that, if $k>1$ then

$$
{ }_{i} A_{2 k, 2 l}^{p, q}={ }_{i, i} X_{2 k, 2 l+1}^{p, q}=-{ }_{i, i} X_{2 l, 2 k-1}^{q, p}=-{ }_{i} A_{2 l, 2 k-2}^{q, p} \text {. }
$$

Therefore, if $k>1$ then

$$
{ }_{i} A_{2 k, 2 l}=-\left[{ }_{i} A_{2 l, 2 k-2}\right]^{\prime}
$$

But from Theorem 6.15 we know $(i) V /(i-1) V$ is the direct sum of its $F G$-submodules $Q_{2 k}^{i}$, so it follows from (50) that $\phi$ is completely determined by its restrictions to

$$
Q_{2}^{i} \times Q_{2}^{i}, Q_{2}^{i} \times Q_{4}^{i}, \ldots, Q_{2}^{i} \times Q_{2 s_{i}}^{i}
$$


Since (36) is a monomorphism, it follows from above that (37) is also a monomorphism.

Suppose next that $1 \leq i<j \leq \mathbf{t}$ and $\left(\phi_{i}, \phi_{j}\right) \in \operatorname{Bil}((i) V /(i-1) V,(j) V /(j-1) V) \oplus$ $\operatorname{Bil}((j) V /(j-1) V,(i) V /(i-1) V)$ belongs to the image of (40). From the very definition of $S$ we see that $\left(\phi_{1}, \phi_{2}\right)$ is the image under (39) of $\widehat{\varphi_{g}} \in \mathcal{M}_{i}$ for some $g \in G\left[{ }^{\infty} V\right]$. For $1 \leq k \leq s_{i}$ and $1 \leq l \leq s_{j}$, let ${ }_{i, j} A_{2 k, 2 l} \in M_{m_{i}, m_{j}}(F)$ and ${ }_{j, i} A_{2 l, 2 k} \in M_{m_{j}, m_{i}}(F)$ denote the Gram matrices of $\left.\phi_{1}\right|_{Q_{2 k}^{i} \times Q_{2 l}^{j}}$ and $\left.\phi_{2}\right|_{Q_{2 l}^{j} \times Q_{2 k}^{i}}$ relative to the bases of $Q_{2 k}^{i}$ and $Q_{2 l}^{i}$ described in Theorem 6.15. Reasoning as above, we deduce that, if $k>1$ then

$$
{ }_{i, j} A_{2 k, 2 l}=-\left[{ }_{j, i} A_{2 l, 2 k-2}\right]^{\prime}
$$

As above, this implies that the pair $\left(\phi_{1}, \phi_{2}\right)$ is completely determined by the restrictions of $\phi_{1}$ to

$$
Q_{2}^{i} \times Q_{2}^{j}, Q_{2}^{i} \times Q_{4}^{j}, \ldots, Q_{2}^{i} \times Q_{2 s_{j}}^{j}
$$

and restrictions of $\phi_{2}$ to

$$
Q_{2}^{j} \times Q_{2}^{i}, Q_{2}^{j} \times Q_{4}^{i}, \ldots, Q_{2}^{j} \times Q_{2 s_{i}}^{i}
$$

Since (40) is a monomorphism, it follows from above that (41) is also a monomorphism.

By collecting all monomorphisms (37) and (41), and applying them to the series for $S$ produced after Definition 10.17, we obtain the inequality

$$
\operatorname{dim} S \leq \sum_{1 \leq i \leq \mathbf{t}} s_{i} m_{i}^{2}+\sum_{1 \leq i \neq j \leq \mathbf{t}} s_{j} m_{i} m_{j}+s_{i} m_{j} m_{i}
$$

that is

$$
\operatorname{dim} S \leq\left(s_{1} m_{1}+\cdots+s_{\mathbf{t}} m_{\mathbf{t}}\right)\left(m_{1}+\cdots+m_{\mathbf{t}}\right)=\left(\operatorname{dim} V /{ }^{\infty} V\right)\left(m_{1}+\cdots+m_{\mathbf{t}}\right)
$$

By combining the inequalities (43) and (51) we deduce the equality (42) and the fact that all maps (37) and (41) are isomorphisms. The remaining assertions of the theorem are now consequence of Theorem 6.15.

\subsection{Dimension of $G\left[{ }^{\infty} V / V_{\infty}\right] \cap G\left[V_{\infty}\right] / G\left[{ }^{\infty} V\right]$}

Recall the $F$-vector space decomposition $V=V_{\text {odd }} \oplus\left(V_{\text {even }} \oplus V_{\text {ndeg }}\right) \oplus V_{\text {odd }}^{\dagger}$, and consider a basis of $V$ formed by putting together, one after another, bases of the 3 summands in 
the above decomposition. We shall identify each element of $G\left[V_{\infty}\right] \cap G\left[{ }^{\infty} V / V_{\infty}\right]$ with its matrix. The Gram matrix $A$ of $\varphi$ has the form

$$
A=\left(\begin{array}{ccc}
0 & 0 & A_{1} \\
0 & A_{2} & 0 \\
A_{3} & 0 & 0
\end{array}\right) .
$$

By Lemma 3.1 if $X \in G\left[V_{\infty}\right] \cap G\left[{ }^{\infty} V / V_{\infty}\right]$ then

$$
X=\left(\begin{array}{ccc}
1 & Y_{1} & Z \\
0 & 1 & Y_{2} \\
0 & 0 & 1
\end{array}\right) .
$$

The equation $X^{\prime} A X=A$ defining $G$ translates into

$$
\begin{gathered}
Y_{1}^{\prime} A_{1}+A_{2} Y_{2}=0, \\
Y_{2}^{\prime} A_{2}+A_{3} Y_{1}=0, \\
Z^{\prime} A_{1}+A_{3} Z+Y_{2}^{\prime} A_{2} Y_{2}=0 .
\end{gathered}
$$

By Lemma 3.2 the conditions for $X$ to belong to $\left.G{ }^{\infty} V\right]$ are $Y_{1}=0, Y_{2}=0$ and (54).

10.19 Lemma The group $G\left[V_{\infty}\right] \cap G\left[{ }^{\infty} V / V_{\infty}\right] / G\left[{ }^{\infty} V\right]$ is isomorphic to the $F$-vector space $Y$ of all pairs $\left(Y_{1}, Y_{2}\right)$ satisfying (52) and (53).

Proof. Using the above notation we define the map $\gamma: G\left[V_{\infty}\right] \cap G\left[{ }^{\infty} V / V_{\infty}\right] \rightarrow Y$ given by $X \mapsto\left(Y_{1}, Y_{2}\right)$. One easily verify that $\gamma$ is a group homomorphism with kernel $G\left[{ }^{\infty} V\right]$. It remains to show that $\gamma$ is surjective. Consider the linear map $\delta: \operatorname{Hom}_{F}\left(V_{\text {odd }}^{\dagger}, V_{\infty}\right) \rightarrow$ $\operatorname{End}_{F}\left(V_{\text {odd }}^{\dagger}\right)$, which in matrix terms is given by $Z \mapsto Z^{\prime} A_{1}+A_{3} Z$. By what we mentioned above, the kernel of $\delta$ is isomorphic to $G\left[{ }^{\infty} V\right]$, which by Theorem 10.18 has dimension $\operatorname{dim}\left(V_{\text {odd }}^{\dagger}\right) \times\left(m_{1}+\cdots+m_{t}\right)$. It follows that the image of $\delta$ has dimension

$\operatorname{dim}\left(V_{\text {odd }}^{\dagger}\right) \times \operatorname{dim}\left(V_{\infty}\right)-\operatorname{dim}\left(V_{\text {odd }}^{\dagger}\right) \times\left(m_{1}+\cdots+m_{t}\right)=\operatorname{dim}\left(V_{\text {odd }}^{\dagger}\right) \times\left[\operatorname{dim}\left(V_{\infty}\right)-\left(m_{1}+\cdots+m_{t}\right)\right]$ and this equals $\operatorname{dim}\left(V_{\text {odd }}^{\dagger}\right) \times \operatorname{dim}\left(V_{\text {odd }}^{\dagger}\right)=\operatorname{dim} \operatorname{End}_{F}\left(V_{\text {odd }}^{\dagger}\right)$. Thus $\delta$ is surjective, whence $\gamma$ must be surjective as well. 
10.20 Proposition The dimension of the $F$-vector space $G\left[V_{\infty}\right] \cap G\left[{ }^{\infty} V / V_{\infty}\right] / G\left[{ }^{\infty} V\right]$ is equal to $\operatorname{dim}\left(V_{\text {even }} \oplus V_{\text {ndeg }}\right) \times\left(m_{1}+\cdots+m_{t}\right)$.

Proof. By making use to Lemma 10.19 one verifies by direct computation that any orthogonal direct decomposition of $V_{\text {even }} \oplus V_{\text {ndeg }}$ resp. $V_{\text {odd }}$ yields a corresponding direct product decomposition of $F$-vector space $G\left[V_{\infty}\right] \cap G\left[{ }^{\infty} V / V_{\infty}\right] / G\left[^{\infty} V\right]$. Hence we are reduced to prove this result when both bilinear spaces $V_{\text {even }} \oplus V_{\text {ndeg }}$ and $V_{\text {odd }}$ are indecomposable. Thus $V_{\text {odd }}$ has a basis $e_{1}, \ldots, e_{2 s+1}$ relative to which the Gram matrix of $\varphi$ is equal to $J_{2 s+1}(0)$ and there are two cases to be considered.

CASE I: $V_{\text {even }}=(0)$ (there is no need to assume that $V_{\text {ndeg }}$ is indecomposable).

Let $f_{1}, \ldots, f_{n}$ be a basis of $V_{\text {ndeg }}$ and let $g \in \mathrm{GL}(V)$. Suppose that

$g e_{1}=e_{1}, g e_{2}=e_{2}+u_{2}+v_{2}, g e_{3}=e_{3}, g e_{4}=e_{4}+u_{4}+v_{4}, \ldots, g_{2 s}=e_{2 s}+u_{2 s}+v_{2 s}, g e_{2 s+1}=e_{2 s+1}$

and

$g f_{1}=f_{1}+a_{1,1} e_{1}+a_{1,3} e_{3}+\cdots+a_{1,2 s+1} e_{2 s+1}, \ldots, g f_{n}=f_{n}+a_{n, 1} e_{1}+a_{n, 3} e_{3}+\cdots+a_{n, 2 s+1} e_{2 s+1}$,

for some $a_{i j} \in F, u_{2 k} \in V_{\text {ndeg }}$ and $v_{2 l} \in V_{\infty}$.

We claim that given any choice of $a_{1,1}, \ldots, a_{n, 1}$ we can find $u_{2 k} \in V_{\text {ndeg }}, v_{2 l} \in V_{\infty}$ and all other $a_{i j} \in F$ such that $g \in G\left[V_{\infty}\right] \cap G\left[{ }^{\infty} V / V_{\infty}\right]$, and, moreover, $g G\left[{ }^{\infty} V\right]$ will be unique.

It suffices to find $u_{2 k} \in V_{\text {ndeg }}$ and all other $a_{i j} \in F$ so that $g e_{2}, \ldots, g e_{2 s}$ remain orthogonal to $g f_{1}, \ldots, g f_{n}$, and show that the choices for these are unique. Indeed, the proof of Lemma 10.19 explains why the $v_{2 l}$ will then exist to form $g \in G\left[V_{\infty}\right] \cap G\left[{ }^{\infty} V / V_{\infty}\right]$, and it is clear that $g G\left[^{\infty} V\right]$ will then be unique.

In order to find the unique $u_{2 k} \in V_{\text {ndeg }}$ and $a_{i j} \in F, j>1$, note first that $\varphi\left(g e_{2}, g f_{l}\right)=0$ translates into $\varphi\left(u_{2}, f_{l}\right)=-a_{l 1}, 1 \leq l \leq n$. As the restriction of $\varphi$ to $V_{\text {ndeg }}$ is nondegenerate, $u_{2}$ exists and is unique. Secondly $\varphi\left(g f_{l}, g e_{2}\right)=0$, translates into $a_{l 3}=$ $-\varphi\left(f_{l}, u_{2}\right)$, so all $a_{l 3}, 1 \leq l \leq n$, exist and are unique. We may now repeat this procedure to determine $u_{4}$ and then all $a_{l 5}$ in a unique manner, etc.

CASE II: $V_{\text {ndeg }}=(0)$ and $V_{\text {even }}$ has a basis $f_{1}, f_{2}, \ldots, f_{2 n-1}, f_{2 n}$ relative to which the matrix of $\varphi$ is equal to $J_{2 n}(0)$. 
We first consider a family of $2 n$ 1-parameter subgroups of $G\left[V_{\infty}\right] \cap G\left[{ }^{\infty} V / V_{\infty}\right]$. It will be obvious from the definition that non-identity members of different 1-parameter subgroups are linearly independent modulo $G\left[{ }^{\infty} V\right]$. Our family is naturally divided into two subfamilies, say $\gamma$ and $\delta$, each of them consisting of $n$ 1-parameter subgroups. The $\gamma$ family consists of $\gamma_{1, a}, \gamma_{3, b}, \ldots, \gamma_{2 n-1, z} \in G\left[V_{\infty}\right] \cap G\left[{ }^{\infty} V / V_{\infty}\right]$, where $a, b, \ldots, z \in F$, all of which fix $R^{\infty}\left(V_{\text {even }}\right)=\left(f_{2}, \ldots, f_{2 n}\right)$ pointwise, and the $\delta$ family consists of $\delta_{2 n, a}, \gamma_{2 n-2, b}, \ldots, \gamma_{2, z} \in$ $G\left[V_{\infty}\right] \cap G\left[{ }^{\infty} V / V_{\infty}\right]$, where $a, b, \ldots, z \in F$, all of which fix $L^{\infty}\left(V_{\text {even }}\right)=\left(f_{1}, \ldots, f_{2 n-1}\right)$. As elements of $G\left[V_{\infty}\right] \cap G\left[{ }^{\infty} V / V_{\infty}\right]$ they all fix $V_{\infty}=\left(e_{1}, e_{3}, \ldots, e_{2 s+1}\right)$ pointwise. In the $\gamma$ family we have

$$
\begin{gathered}
\gamma_{1, a} f_{1}=f_{1}+a e_{1}, \gamma_{1, a} e_{2}=e_{2}-a f_{2}, \gamma_{1, a} f_{3}=f_{3}+a e_{3}, \gamma_{1, a} e_{4}=e_{4}-a f_{4}, \ldots \\
\gamma_{3, a} f_{1}=f_{1}, \gamma_{3, a} f_{3}=f_{3}+a e_{1}, \gamma_{3, a} e_{2}=e_{2}-a f_{4}, \gamma_{3, a} f_{5}=f_{5}+a e_{3}, \gamma_{3, a} e_{4}=e_{4}-a f_{6}, \ldots
\end{gathered}
$$

with the next $\gamma_{i, a}$ similarly defined until

$$
\gamma_{2 n-1, a} f_{1}=f_{1}, \ldots, \gamma_{2 n-1, a} f_{2 n-3}=f_{2 n-3}, \gamma_{2 n-1, a} f_{2 n-1}=f_{2 n-1}+a e_{1}
$$

and

$$
\gamma_{2 n-1, a} e_{2}=e_{2}-a f_{2 n}, \gamma_{2 n-1, a} e_{4}=e_{4}, \ldots, \gamma_{2 n-1, a} e_{2 s}=e_{2 s}
$$

In the $\delta$ family the first member is defined by

$$
\delta_{2 n, a} f_{2 n}=f_{2 n}+a e_{2 s+1}, \gamma_{2 n, a} e_{2 s}=e_{2 s}-a f_{2 n-1},
$$

and

$$
\delta_{2 n, a} f_{2 n-2}=f_{2 n-2}+a e_{2 s-1}, \gamma_{2 n, a} e_{2 s-2}=e_{2 s-2}-a f_{2 n-3}, \ldots
$$

the second member by

$$
\delta_{2 n-2, a} f_{2 n}=f_{2 n}, \delta_{2 n-2, a} f_{2 n-2}=f_{2 n-2}+a e_{2 s+1}, \gamma_{2 n-2, a} e_{2 s}=e_{2 s}-a f_{2 n-3},
$$

and

$$
\delta_{2 n-2, a} f_{2 n-4}=f_{2 n-4}+a e_{2 s-1}, \gamma_{2 n-2, a} e_{2 s-2}=e_{2 s-2}-a f_{2 n-5}, \ldots,
$$

with the next $\delta_{i, a}$ similarly defined until

$\delta_{2, a} f_{2 n}=f_{2 n}, \ldots, \delta_{2, a} f_{4}=f_{4}, \delta_{2, a} f_{2}=f_{2}+a e_{2 s+1}, \delta_{2, a} e_{2 s}-a f_{1}, \delta_{2, a} e_{2 s-2}=e_{2 s-2}, \ldots, \delta_{2, a} e_{2}=e_{2}$ 
This explicit family of $2 n$ 1-parameter subgroups of $G\left[V_{\infty}\right] \cap G\left[{ }^{\infty} V / V_{\infty}\right]$ show that the dimension of $G\left[V_{\infty}\right] \cap G\left[{ }^{\infty} V / V_{\infty}\right] / G\left[{ }^{\infty} V\right]$ is at least $2 n$. We next show the reverse inequality. For this purpose we consider the bilinear space $W=L^{2}(V) / L(V)$, whose bilinear form is the one naturally induced by $\varphi$ (this works since $L(V)$ is contained in radical of $L^{2}(V)$ ). The canonical form-preserving linear map $V \rightarrow W$ induces a canonical group homomorphism $G(V) \rightarrow G(W)=P$. The latter maps $V_{\infty}$ into $W_{\infty}$ and ${ }^{\infty} V$ into ${ }^{\infty} W$, thereby yielding a group homomorphism, actually a linear map from $G\left[V_{\infty}\right] \cap G\left[{ }^{\infty} V / V_{\infty}\right] / G\left[{ }^{\infty} V\right]$ into $P\left[W_{\infty}\right] \cap P\left[{ }^{\infty} W / W_{\infty}\right]$. One verifies that the kernel of this map is generated by the classes modulo $G\left[^{\infty} V\right]$ of $\gamma_{2 n-1, a}$ and $\delta_{2, b}$ as $a, b$ run through $F$, so it has dimension 2 . Applying this procedure repeatedly until $\operatorname{dim} W_{\text {odd }}=1$ or $W_{\text {even }}=0$-in which cases our result is obvious- it follows that $\operatorname{dim} G\left[V_{\infty}\right] \cap G\left[{ }^{\infty} V / V_{\infty}\right] \leq 2 n$, as required.

As a corollary of Theorem 10.18 and Proposition 10.20 we finally obtain

\subsection{Theorem $\operatorname{dim} G\left[V_{\infty}\right] \cap G\left[{ }^{\infty} V / V_{\infty}\right]=\operatorname{dim}\left(V / V_{\infty}\right) \times\left(m_{1}+\cdots+m_{t}\right)$}

We know from Lemma 10.2 that $G\left[V_{\infty}\right] \cap G\left[^{\infty} V / V_{\infty}\right]$ is a nilpotent group of class $\leq 2$. The following result describes the exact nilpotency class. The proof, which will be omitted, consists of a case by case analysis, all of which is direct consequence of the preceding material. We make however one clarifying remark: if $V_{\text {odd }} \neq \operatorname{Rad}(V)$ and $V_{\text {even }} \neq(0)$ then the elements $\gamma_{1, a}$ and $\delta_{2, b}$ of $G\left[V_{\infty}\right] \cap G\left[{ }^{\infty} V / V_{\infty}\right]$ do not commute provided $a, b \in F$ are non-zero.

10.22 Lemma (a) If $V_{\text {odd }}=(0)$ or $V=V_{\text {odd }}$ then $G\left[V_{\infty}\right] \cap G\left[{ }^{\infty} V / V_{\infty}\right]$ is trivial.

(b) If $\left(V_{\text {odd }} \neq(0)\right.$ and $\left.V \neq V_{\text {odd }}\right)$ and $\left[\left(V_{\text {odd }}=\operatorname{Rad}(V)\right)\right.$ or $\left(V_{\text {even }}=(0)\right.$ and $V_{\text {odd }}$ has at most one indecomposable block of size $\geq 3$ and $\left.\left.\operatorname{dim} V_{\text {ndeg }}=1\right)\right]$ then $G\left[V_{\infty}\right] \cap G\left[{ }^{\infty} V / V_{\infty}\right]$ is non-trivial and abelian.

(c) In all other cases $G\left[V_{\infty}\right] \cap G\left[{ }^{\infty} V / V_{\infty}\right]$ is non-abelian. 


\section{Decomposing $G(V)$ in terms of $G\left(V_{\text {odd }}\right), G\left(V_{\text {even }}\right)$ and $G\left(V_{\text {ndeg }}\right)$}

The next result summarizes what we know about the $G\left(V_{\text {odd }}\right)$. A notable fact is that even though $V_{\text {odd }}$ is far from being uniquely determined by $V$, the image of the restriction group homomorphism $G\left(V_{\text {odd }}\right) \rightarrow G L\left(V_{\infty}\right)$ is the same for all choices of $V_{\text {odd }}$, as it coincides with the image of $G \rightarrow G L\left(V_{\infty}\right)$.

\subsection{Theorem We have}

$$
G\left(V_{\text {odd }}\right)=G\left[{ }^{\infty} V\right] \rtimes G\left(V_{\text {odd }}\right) \cap G\left\{V_{\text {odd }}^{\dagger}\right\}=G\left[^{\infty} V\right] \rtimes(U \rtimes E),
$$

where the action of $E \cong \prod_{1 \leq i \leq t} \mathrm{GL}_{m_{i}}(F)$ on the unipotent group $U$, and the action of $U \rtimes E$ on the abelian unipotent group $\left.G^{\infty} V\right]$ possess the properties previously described in the paper.

Moreover, the restriction maps $G\left(V_{\text {odd }}\right) \rightarrow \mathrm{GL}\left(V_{\infty}\right)$ and $G \rightarrow \mathrm{GL}\left(V_{\infty}\right)$ have exactly the same image, say $H$. Indeed, both maps restricted to $U \rtimes E$ yield the isomorphism $U \rtimes E \rightarrow H$, while both maps have split kernels, respectively equal to $G\left[{ }^{\infty} V\right]$ and $G\left[V_{\infty}\right]$. Thus $H$ is isomorphic to

$$
G\left(V_{\text {odd }}\right) / G\left[{ }^{\infty} V\right] \cong U \rtimes E \cong G / G\left[V_{\infty}\right]
$$

Proof. Applying Lemma 3.2 to the decomposition (25) with $V=V_{\text {odd }}$ and making use of (24) we get (55). Again by Lemma 3.2, the restriction map $G\left(V_{\text {odd }}\right) \rightarrow \operatorname{GL}\left(V_{\infty}\right)$ has $G\left[{ }^{\infty} V\right]$ in its kernel. Let $H$ denote its image. By Lemma $3.1 G\left[V_{\infty}\right] \cap G\left(V_{\text {odd }}\right) \cap G\left\{V_{\text {odd }}^{\dagger}\right\}=<1>$, whence $U \rtimes E \rightarrow H$ is an isomorphism. It follows from (25) that the image of $G \rightarrow \operatorname{GL}\left(V_{\infty}\right)$ coincides with the image of $U \rtimes E \rightarrow \mathrm{GL}\left(V_{\infty}\right)$, that is $H$. This completes the proof.

Next we produce further decompositions for $G$.

11.2 Theorem We have the following decompositions for $G(V)$.

$$
G(V)=\left(G\left[^{\infty} V / V_{\infty}\right] \cap G\left[V_{\infty}\right]\right)\left(G\left(V_{\text {odd }}\right) \times G\left(V_{\text {even }}\right) \times G\left(V_{\text {ndeg }}\right)\right),
$$

where the intersection of $G\left(V_{\text {odd }}\right) \times G\left(V_{\text {even }}\right) \times G\left(V_{\text {ndeg }}\right)$ with the normal subgroup $G\left[{ }^{\infty} V / V_{\infty}\right] \cap$ $G\left[V_{\infty}\right]$ of $G(V)$ is the normal subgroup $G\left[{ }^{\infty} V\right]$ of $G(V)$;

$$
G(V)=G\left[{ }^{\infty} V / V_{\infty}\right] \cap G\left[V_{\infty}\right] \rtimes\left(\left(G\left(V_{\text {odd }}\right) \cap G\left\{V_{\text {odd }}^{\dagger}\right\}\right) \times G\left(V_{\text {even }}\right) \times G\left(V_{\text {ndeg }}\right)\right),
$$


where $G\left(V_{\text {odd }}\right) / G\left[{ }^{\infty} V\right] \cong G\left(V_{\text {odd }}\right) \cap G\left\{V_{\text {odd }}^{\dagger}\right\}$;

$$
G(V)=G\left[V_{\infty}\right] G\left(V_{\text {odd }}\right)
$$

where $G\left[V_{\infty}\right] \cap G\left(V_{\text {odd }}\right)=G\left[{ }^{\infty} V\right]$

$$
G=G\left[V_{\infty}\right] G\left[{ }^{\infty} V / V_{\infty}\right]
$$

where $G\left[V_{\infty}\right] \cap G\left[{ }^{\infty} V / V_{\infty}\right]$ is a unipotent normal subgroup of $G$ with nilpotency class $\leq 2$.

Proof. The first three decompositions follow from Theorems 7.1 and 11.1, while the fourth follows from the third.

Finally we consider the special but interesting case when $V=V_{\text {odd }}$ is homogenous, namely when $V=V_{\text {odd }}$ is the direct sum of $m$ Gabriel blocks of equal size $2 s+1$. The isomorphism type of $G$ is fully revealed in this case.

11.3 Theorem Suppose $V=V_{\text {odd }}$ is the direct sum of $m$ Gabriel blocks of size $2 s+1$. Then

$$
G \cong\left(\prod_{1 \leq k \leq s} M_{m}(F)\right) \rtimes \mathrm{GL}_{m}(F),
$$

where $\mathrm{GL}_{m}(F)$ acts diagonally on $\prod_{1 \leq k \leq s} M_{m}(F)$ by congruence.

Internally, $G\left[V_{\infty}\right]$ has a natural structure of $F G$-module of dimension $s m^{2}$ over $F$. As a module over $\mathrm{GL}_{m}(F) \cong G / G\left[V_{\infty}\right], G\left[V_{\infty}\right]$ is isomorphic to $\prod_{1 \leq k \leq s} M_{m}(F)$, upon which $\mathrm{GL}_{m}(F)$ acts diagonally by congruence.

Proof. Observe first of all that $N=G\left[V_{\infty}\right]=G\left[{ }^{\infty} V\right]$, so $G=G\left[{ }^{\infty} V\right] \rtimes E$, where $E \cong$ $\mathrm{GL}_{m}(F)$ and the action of $E$ on $G\left[^{\infty} V\right]$ has been determined. More precisely, as the case $s=0$ is obvious, we may assume that $s \geq 1$. Since $t=1$ and $\operatorname{Rad}(V)=0$, Theorem 10.18 yields

$$
G\left[^{\infty} V\right]=G\left[^{\infty} V\right] \cap G[V / \operatorname{Rad}(V)] \cong S \cong \underset{1 \leq k \leq s}{\oplus} \operatorname{Bil}\left(Q_{2}^{1}, Q_{2 k}^{1}\right),
$$

and the indicated action of $E \cong M_{m}(F)$ on $\underset{1 \leq k \leq s}{\oplus} \operatorname{Bil}\left(Q_{2}^{1}, Q_{2 k}^{1}\right) \cong \prod_{1 \leq k \leq s} M_{m}(F)$. 


\section{Inductive approach}

It is possible to extract useful information on $G$ by studying the canonical group homomorphism

$$
G(V) \rightarrow G\left(L^{2}(V) / L^{1}(V)\right)
$$

Here the bilinear space $L^{2}(V) / L^{1}(V)$ can be obtained from $V$ in a straightforward manner: its non-degenerate parts are equivalent, and all Gabriel blocks of $V$ decrease in size by 2 when passing from $V$ to $L^{2}(V) / L^{1}(V)$, except for those of size $\leq 2$ which disappear. The above map is very likely to be surjective (we have checked this in a few cases), so repeated application of it would yield $G$ as constructed from $G\left(V_{\text {ndeg }}\right)$ and the various kernels, all of which respond to the same pattern.

This sort of approach seems to be applicable to $G\left(V_{\text {ndeg }}\right)$, once it is already decomposed as in (28). There is a canonical $G$-invariant filtration for $V_{\text {ndeg }}$ and one produces from it a non-degenerate bilinear space as a section of $V_{\text {ndeg }}$. Special cases have revealed the associated group homomorphism to be surjective as well.

\section{References}

[1] B. Corbas and G.D. Williams, Bilinear forms over an algebraically closed field, J. Pure Appl. Algebra, 165 (2001), 255-266.

[2] J. Dieudonné, La géométrie des groupes classiques, Troisième édition, Ergebnisse der Mathematik und ihrer Grenzgebiete, Band 5, Springer-Verlag, Berlin-New York, 1971.

[3] D.Ž. Đoković and F. Szechtman, Characterization of bilinear spaces with unimodular isometry group, Proc. Amer. Math, Soc., 133 (2005), 2853-2863.

[4] J. Fulman and R. Guralnick, Conjugacy class properties of the extension of $\operatorname{GL}(n, q)$ generated by the inverse transpose involution, J. Algebra 275 (2004), 356-396.

[5] P. Gabriel, Appendix: Degenerate bilinear forms, J. Algebra, 31 (1974), 67-72. 
[6] J.C. Jantzen, Nilpotent Orbits in Representation Theory, in Lie Theory, Lie Algebras and Representations, Progress in Mathematics 228, Eds. J.-Ph. Anker and B. Orsted, Birkäuser, Boston, 2003.

[7] C. Riehm, The equivalence of bilinear forms, J. Algebra, 31 (1974), 44-66.

[8] A.J. Hahn and O.T. O'Meara, The classical groups and K-theory, Grundlehren der Mathematischen Wissenschaften, 291. Springer-Verlag, Berlin, 1989.

[9] R.A. Horn and V.V. Sergeichuk, Invited talk (delivered by the first author) at the International Conference on Matrix Theory and its Applications, Fort Lauderdale, Florida, December 2003.

[10] T.A. Springer and R. Steinberg, Conjugacy classes, Seminar in algebraic groups and related finite groups, Lecture Notes in Mathematics, Springer-Verlag 131 (1970), $167-266$.

[11] G.E. Wall, On the conjugacy classes in the unitary, symplectic and orthogonal groups, J. Austral. Math. Soc. 3, (1963), 1-62.

[12] W.C. Waterhouse, The number of congruence classes in $M_{n}\left(\mathbf{F}_{q}\right)$, Finite Fields and their Applications, 1 (1995), 57-63. 